\title{
MHD FREE CONVECTION-RADIATION INTERACTION IN A POROUS MEDIUM - PART I: NUMERICAL INVESTIGATION
}

\author{
B. VASU \\ Department of Mathematics, Motilal Nehru National Institute of Technology \\ Allahabad - 211004, INDIA \\ R.S.R. GORLA* \\ Department of Mechanical Engineering, Cleveland State University \\ Ohio, 44115, USA \\ E-mail: r.gorla@yahoo.com \\ P.V.S.N. MURTHY \\ Department of Mathematics, Indian Institute of Technology \\ Kharagpur- 721 302, INDIA \\ V.R. PRASAD \\ Department of Mathematics, Vellore Institute of Technology, Vellore- 632014, INDIA \\ O.ANWAR BÉG
}

Department of Mechanical and Aeronautical Engineering, School of Science, Engineering and Environment (SEE), Newton building, Salford University, Manchester, M54WT, UK

\author{
S. SIDDIQA \\ Department of Mathematics \\ COMSATS Institute of Information Technology \\ Attock, PAKISTAN
}

\begin{abstract}
A numerical investigation of two dimensional steady magnetohydrodynamics heat and mass transfer by laminar free convection from a radiative horizontal circular cylinder in a non-Darcy porous medium is presented by taking into account the Soret/Dufour effects. The boundary layer conservation equations, which are parabolic in nature, are normalized into non-similar form and then solved numerically with the well-tested, efficient, implicit, stable Keller-Box finite-difference scheme. We use simple central difference derivatives and averages at the mid points of net rectangles to get finite difference equations with a second order truncation error. We have conducted a grid sensitivity and time calculation of the solution execution. Numerical results are obtained for the velocity, temperature and concentration distributions, as well as the local skin friction, Nusselt number and Sherwood number for several values of the parameters. The dependency of the thermophysical properties has been discussed on the parameters and shown graphically. The Darcy number accelerates the flow due to a corresponding rise in permeability of the regime and concomitant decrease in Darcian impedance. A comparative study between the previously published and present results in a limiting sense is found in an excellent agreement.
\end{abstract}

Key words: implicit finite difference scheme, Keller-Box method, non-Darcy porous media transport, magnetic field; horizontal circular cylinder.

\section{Introduction}

During the past decades there has been an increased interest in solving systems of nonlinear differential equations associated with physical problems. Most of the problems arising in science and engineering are

\footnotetext{
* To whom correspondence should be addressed
} 
nonlinear. They are naturally difficult to solve. Traditional analytical approximations are valid only for weakly nonlinear problems, and often break down for problems with strong nonlinearity. However, remarkable progress has been made in developing new and powerful techniques for solving the nonlinear differential equations, particularly in the fields of fluid mechanics, biology, aerospace engineering, chemical engineering, etc. Due to the difficulties of the problems researchers frequently pursue to obtain numerical solutions to a nonlinear problem. One such technique, which has shown a great potential over the past few years, is the Keller-box method. An alternative implicit method due to Keller is now described and is referred to as the Box method. This method has several very desirable features that make it appropriate for the solution of all parabolic partial differential equations. The main features of this method are:

- only slightly more arithmetic to solve than the Crank-Nicolson method,

- $\quad$ second order accuracy with arbitrary (non-uniform) $x$ and $y$ spacing,

- allows very rapid variations,

- allows easy programming of the solution of large numbers of coupled equations.

This method, originally developed for low speed aerodynamic boundary layers by Keller [1] has been employed in a diverse range of nonlinear magneto-hydrodynamics and coupled heat transfer problems. These include magnetic boundary layers (Chiam [2]), wavy thermal boundary layers [Rees and Pop [3]], rotating hydro-magnetic convection (Hossain et al. [4]), thermal convection in porous regimes (Rees and Hossain [5]), magneto-viscoelastic heat transfer in porous media (Bég et al.[6]), radiation-convection viscoelastic boundary layers (Bég et al. [7]), hydro-magnetic convection from an elastic cylinder (Ishak et al. [8]) and hydro-magnetic thermophoretic mixed convection in porous media (Damseh et al. [9]), heat and mass transfer in micropolar regime (Bég et al. [10]), radiative-convective porous media flows (Prasad et al. [11]). Recently, Gorla and Vasu [12] and Gorla et al. [13] have studied an unsteady convective heat transfer in a non-Newtonian nanofluid.

This method has been found to be efficient and flexible in dealing with the problems of boundary layer flows. This method is chosen since it seems to be the most flexible of the common methods, being easily adaptable for solving equations of any order.

Transport phenomena in porous media constitute numerous important flow regimes in many branches of engineering and applied physics. The vast majority of models have considered isotropic, homogenous porous media, usually employing the Darcy law, which is valid for low velocity, viscous-dominated transport. However, porous media are generally heterogeneous and exhibit variable porosity. An early study of flow through variable porosity media was conducted by Roblee et al. [14] for the case of radial variation in chemical engineering systems. Much later a seminal theoretical and experimental study was presented by Vafai [15] who studied the influence of variable porosity and also inertial forces (Forchheimer drag) on thermal convection flow in porous media, with the channelling effect being studied in detail. Zueco et al. [16] used network simulation to investigate the hydromagnetic heat transfer of a micro-structural liquid material in a vertical pipe containing a Darcy-Forchheimer porous medium. An important study of natural convection boundary layers in Darcian porous media was presented by Minkowycz and Cheng [17], although they did not consider the magnetic case. Hamzeh Taha et al. [18]investigated the effect of radiation on magnetohydrodynamic free convection boundary of a solid sphere with Newtonian heating. Kumari and Gorla [19]presented the MHD boundary layer flow past a wedge in a non-Newtonian nanofluid. Kameswaran et al. [20] considered a mixed convection from a wavy surface embedded in a thermally stratified nanofluid saturated porous medium with non-linear Boussinesq approximation. Beg et al. [21] studied numerically the computational modelling of magnetohydrodynamic convection from a rotating cone in orthotropic Darcian porous media. Very recently, Vasu et al. [22] investigated the entropy generation analysis in nonlinear convection flow of thermally stratified fluid in a saturated porous medium with convective boundary condition.

Bég et al. [23] used the local non-similarity method with a shooting procedure to analyze mixed convective heat and mass transfer from an inclined plate with Soret/Dufour effects with applications in solar energy collector systems. Bhargava et al. [24] also studied oscillating hydromagnetic heat and mass transfer with Soret and Dufour effects. El-Kabeir and Chamkha [25] focused on the study of heat and mass transfer by mixed convection over a vertical slender cylinder in the presence of chemical reaction and thermaldiffusion and diffusion-thermo effects. Bhattacharyya et al. [26] presented a mathematical model for the 
Soret and Dufour effects on the convective heat and mass transfer in stagnation-point flow of viscous incompressible fluid towards a shrinking surface.

The objective of the present paper is to investigate the effects of Soret (thermo-diffusion) and Dufour (diffusion-thermal) on the hydromagnetic convective boundary layer on a horizontal permeable cylinder embedded in a non-Darcy porous regime where radiation is included by assuming Rosseland diffusion approximation. An implicit numerical solution is obtained to the transformed boundary layer equations.

\section{Mathematical analysis}

A steady, laminar, two-dimensional, viscous, incompressible, electrically-conducting, buoyancydriven convection boundary layer heat and mass transfer from a horizontal permeable cylinder embedded in a non-Darcy saturated regime with radiation and Soret/Dufour effects is analyzed. A uniform magnetic field $B_{0}$, is applied in the radial direction, i.e. normal to the cylinder surface. Figure 1 shows the flow model and physical coordinate system.

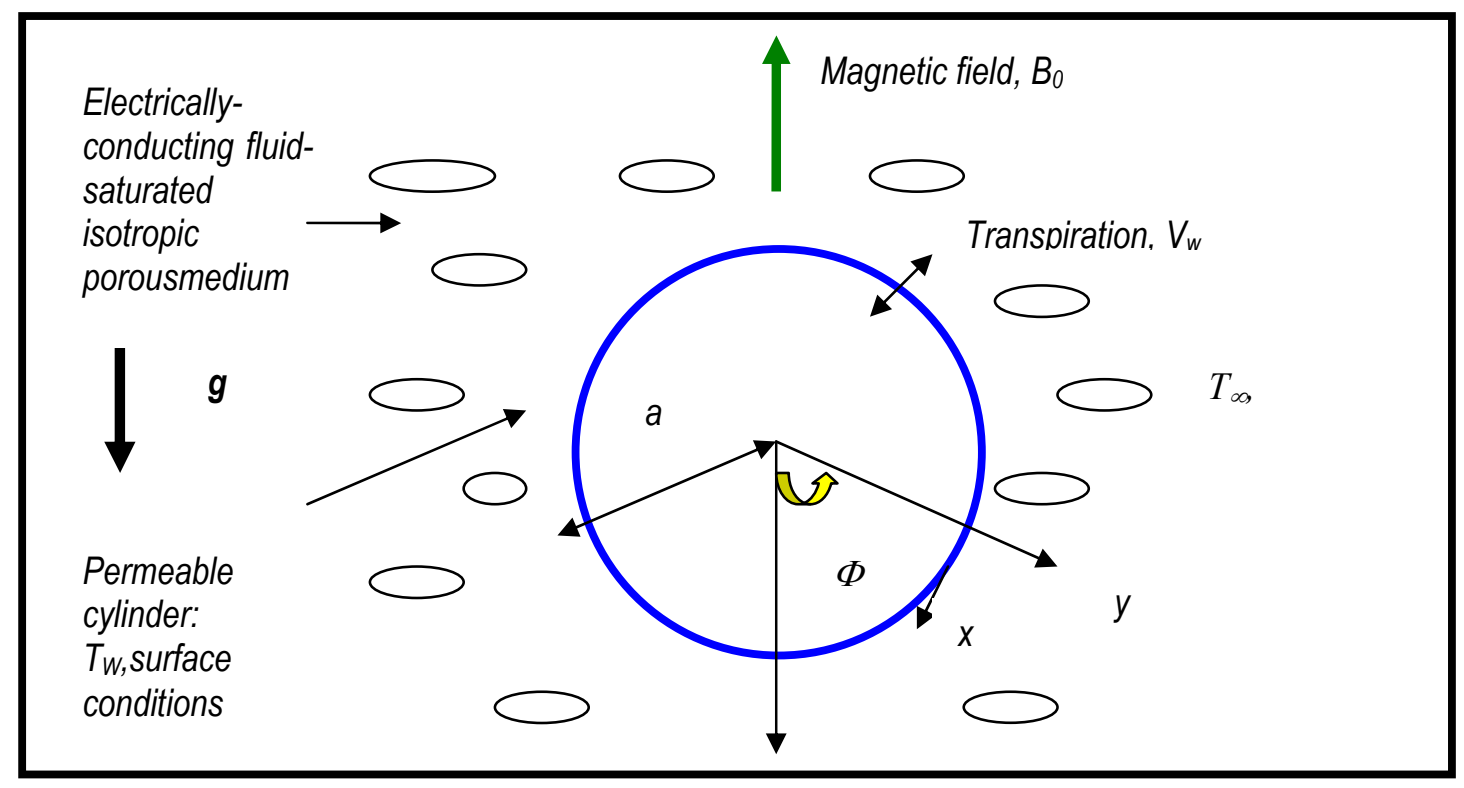

Fig.1. Physical model and coordinate system.

The $x$ - coordinate is measured along the circumference of the horizontal cylinder from the lowest point and the $y$-coordinate is measured normal to the surface, with ' $\mathrm{a}$ ' denoting the radius of the horizontal cylinder. $\Phi=x / a$, is the angle of the $y$ - axis with respect to the vertical $(0 \leq \Phi \leq \pi)$. The gravitational acceleration $g$, acts downwards. The magnetic Reynolds number is assumed to be small enough to neglect magnetic induction effects. Hall current and ion slip effects are also neglected since the magnetic field is weak. We also assume that the Boussinesq approximation holds, i.e. that density variation is only experienced in the buoyancy term in the momentum equation. Additionally, the electron pressure (for weakly conducting fluids) and the thermoelectric pressure are negligible. Both the horizontal cylinder and the fluid are maintained initially at the same temperature and concentration. Instantaneously they are raised to a temperature $T_{w}$ ( $>T_{\infty}$, the ambient temperature of the fluid) and concentration $C_{w}\left(>C_{\infty}\right.$, the far-field concentration) remain unchanged. The fluid properties are assumed to be constant except the density variation in the buoyancy force term. In line with the approach of Yih [27] and introducing the boundary layer approximations, the governing conservation equations can be written as follows 


$$
\begin{aligned}
& \frac{\partial u}{\partial x}+\frac{\partial v}{\partial y}=0 \\
& u \frac{\partial u}{\partial x}+v \frac{\partial u}{\partial y}=g \beta\left(T-T_{\infty}\right) \sin \left(\frac{x}{a}\right)+g \beta^{*}\left(C-C_{\infty}\right) \sin \left(\frac{x}{a}\right)+v \frac{\partial^{2} u}{\partial y^{2}}-\frac{\sigma \beta_{0}^{2}}{\rho} u-\frac{v}{K} u-\Gamma u^{2}, \\
& u \frac{\partial T}{\partial x}+v \frac{\partial T}{\partial y}=\alpha \frac{\partial^{2} T}{\partial y^{2}}-\frac{1}{\rho c_{p}} \frac{\partial q_{r}}{\partial y}+\frac{D_{m} K_{T}}{c_{s} c_{p}} \frac{\partial^{2} C}{\partial y^{2}} \\
& u \frac{\partial C}{\partial x}+v \frac{\partial C}{\partial y}=D_{m} \frac{\partial^{2} C}{\partial y^{2}}+\frac{D_{m} K_{T}}{T_{m}} \frac{\partial^{2} T}{\partial y^{2}} .
\end{aligned}
$$

The boundary conditions are prescribed at the cylinder surface and the edge of the boundary layer regime, respectively as follows

$$
\begin{aligned}
& u=0, \quad v=V_{w}, \quad T=T_{w}, \quad C=C_{w} \quad \text { at } \quad y=0, \\
& u \rightarrow 0, \quad T \rightarrow T_{\infty}, \quad C \rightarrow C_{\infty} \quad \text { as } \quad y \rightarrow \infty
\end{aligned}
$$

where $u$ and $v$ are the velocity components in the $x$ - and $y$-directions, respectively, $K$ and $\Gamma$ - the respective permeability and the inertia coefficient of the porous medium, $v$ is the kinematic viscosity of the conducting fluid, $\beta$ and $\beta^{*}$ - the coefficients of thermal expansion and concentration expansion, respectively, $T$ and $C$ - the temperature and concentration, respectively, $\sigma$ - the electrical conductivity, $B_{0}$ - the externally imposed magnetic field in the $y$-direction, $\rho$ - the density, $D_{m}$ - the mass diffusivity, $c_{p}$ the specific heat capacity, $c_{s}$ - the concentration susceptibility, $\alpha$ - the thermal diffusivity, $T_{m}$ - the mean fluid temperature, $K_{T}$ - the thermal diffusion ratio, $T_{\infty}$ - the free stream temperature, $C_{\infty}$ - the free stream concentration and $V_{w}$ - the uniform blowing/suction velocity. The assumption that the magnetic Reynolds number is small and the induced magnetic field is negligible compared with the applied magnetic field is implicit in the present model. The Hall current is also neglected. It should be noted that in the momentum Eq.(2.2), the fifth term on the right hand side is the porous medium Darcian drag force representing pressure loss due to the presence of the porous medium. The sixth term on the same side is the inertial drag force (also referred to as the Forchheimer impedance) which accounts for additional pressure drop resulting from interpore-mixing appearing at high velocities, as described by Plumb and Huenefeld [28]. The Rosseland diffusion flux model is used and is defined following Modest as follows

$$
q_{r}=-\frac{4 \sigma^{*}}{3 k^{*}} \frac{\partial T^{4}}{\partial y}
$$

where $k^{*}$ is the mean absorption coefficient and $\sigma^{*}$ is the Stefan-Boltzmann constant. Following Raptis and Perdikis [29] we can express the quadratic temperature function in Eq.(2.6) as a linear function of temperature. The Taylor series for $T^{4}$, discarding higher order terms can be shown to give

$$
T^{4} \cong 4 T_{\infty}^{3} T-3 T_{\infty}^{4}
$$


Substitution of this expression into Eq.(2.6) and then the heat conservation Eq.(2.3), eventually leads to the following form of the energy equation

$$
u \frac{\partial T}{\partial x}+v \frac{\partial T}{\partial y}=\frac{1}{\rho c_{p}}\left(k+\frac{16 \sigma^{*} T_{\infty}^{3}}{3 k^{*}}\right) \frac{\partial^{2} T}{\partial y^{2}}+\frac{D_{m} K_{T}}{c_{s} c_{p}} \frac{\partial^{2} C}{\partial y^{2}} .
$$

The stream function $\psi$ is defined by $u=\partial \psi / \partial y$ and $v=-\partial \psi / \partial x$, and therefore, the continuity equation is automatically satisfied. In order to write the governing equations and the boundary conditions in dimensionless form, the following non-dimensional quantities are introduced.

$$
\begin{aligned}
& \xi=\frac{x}{a}, \quad \eta=\frac{y}{a} \sqrt[4]{\mathrm{Gr}}, \quad f(\xi, \eta)=\frac{\psi}{v \xi \sqrt[4]{\mathrm{Gr}}}, \quad \theta(\xi, \eta)=\frac{T-T_{\infty}}{T_{w}-T_{\infty}}, \quad \phi(\xi, \eta)=\frac{C-C_{\infty}}{C_{w}-C_{\infty}}, \\
& \mathrm{Gr}=\frac{g \beta\left(T_{w}-T_{\infty}\right) a^{3}}{v^{2}}, \quad \Lambda=\Gamma a, \quad \mathrm{Da}=\frac{K \sqrt{\mathrm{Gr}}}{a^{2}}, \quad N=\frac{\beta^{*}\left(C-C_{\infty}\right)}{\beta\left(T-T_{\infty}\right)}, \\
& F=\frac{k k_{e}}{4 \sigma^{*} T_{\infty}^{3}}, \quad \operatorname{Pr}=\frac{\rho v c_{p}}{k}, \quad \mathrm{Sc}=\frac{v}{D_{m}}, \quad \mathrm{Du}=\frac{D_{m} K_{T}\left(C_{w}-C_{\infty}\right)}{c_{s} c_{p} v\left(T_{w}-T_{\infty}\right)}, \\
& \mathrm{Sr}=\frac{D_{m} K_{T}\left(T_{w}-T_{\infty}\right)}{v T_{m}\left(C_{w}-C_{\infty}\right)}, \quad M=\sigma B_{0}^{2} a^{2} / \rho v \sqrt{\mathrm{Gr}}, \quad f_{w}=-V_{w} a / v \sqrt[4]{\mathrm{Gr}} .
\end{aligned}
$$

In view of Eq.(2.9), Eqs (2.1), (2.2), (2.8) and (2.4) reduce to the following coupled, nonlinear, dimensionless partial differential equations for momentum, energy and species conservation for the regime

$$
\begin{aligned}
& f^{\prime \prime \prime}+f f^{\prime \prime}-(1+\xi \Lambda) f^{\prime 2}+\frac{\sin \xi}{\xi}(\theta+N \phi)-\left(M+\frac{1}{\mathrm{Da}}\right) f^{\prime}=\xi\left(f^{\prime} \frac{\partial f^{\prime}}{\partial \xi}-f^{\prime \prime} \frac{\partial f}{\partial \xi}\right), \\
& \frac{1}{\operatorname{Pr}}\left(1+\frac{4}{3 F}\right) \theta^{\prime \prime}+f \theta^{\prime}+\operatorname{Du} \phi^{\prime \prime}=\xi\left(f^{\prime} \frac{\partial \theta}{\partial \xi}-\theta^{\prime} \frac{\partial f}{\partial \xi}\right), \\
& \frac{\phi^{\prime \prime}}{\mathrm{Sc}}+f \phi^{\prime}+\operatorname{Sr} \theta^{\prime \prime}=\xi\left(f^{\prime} \frac{\partial \phi}{\partial \xi}-\phi^{\prime} \frac{\partial f}{\partial \xi}\right) .
\end{aligned}
$$

The transformed dimensionless boundary conditions are

$$
\begin{aligned}
& f^{\prime}=0, \quad f=f_{w}, \quad \theta=1, \quad \phi=1 \quad \text { at } \quad \eta=0, \\
& f^{\prime} \rightarrow 0, \quad \theta \rightarrow 0, \quad \phi \rightarrow 0 \quad \text { as } \quad \eta \rightarrow \infty .
\end{aligned}
$$

In the above equations, the primes denote the differentiation with respect to $\eta$, the dimensionless radial coordinate, $\xi$ is the dimensionless tangential coordinate and $\Phi$ the azimuthal coordinate, $\Lambda$ - the local inertia coefficient (Forchheimer parameter), Da - the Darcy parameter, $N$ - concentration to thermal buoyancy ratio parameter, $k$ - thermal conductivity, Pr - the Prandtl number, Sc - the Schmidt number, 
$\mathrm{Du}$ - the Dufour number, $\mathrm{Sr}$ - the Soret number, $M$ - the magnetic parameter, $f_{w}$ - the blowing/suction parameter and $\mathrm{Gr}$ - the Grashof (free convection) parameter, $F$ is the radiation parameter. $f_{w}<0$ for $V_{w}>0$ (the case of blowing), and $f_{w}>0$ for $V_{w}<0$ (the case of suction). Of course, the special case of a solid cylinder surface corresponds to $f_{W}=0$. The engineering design quantities of physical interest include the skin-friction coefficient, Nusselt number and Sherwood number, which are given by

$$
\begin{aligned}
& \frac{1}{2} C_{f} \sqrt[4]{\mathrm{Gr}}=\xi f^{\prime \prime}(\xi, 0), \\
& \frac{\mathrm{Nu}}{\sqrt[4]{\mathrm{Gr}}}=-\theta^{\prime}(\xi, 0) \\
& \frac{\mathrm{Sh}}{\sqrt[4]{\mathrm{Gr}}}=-\phi^{\prime}(\xi, 0)
\end{aligned}
$$

\section{Numerical solution}

In this study an efficient Keller-Box implicit difference method has been employed to solve the nonsimilar, nonlinear parabolic partial differential Eqs (2.10) to (2.12) with boundary conditions (2.13) numerically described in the book by Cebeci and Bradshaw [30]. The basic idea of the Keller-Box method is to write the governing equations in the form of a system of first order equations. We use simple central difference derivatives and averages at the mid points of net rectangles to get finite difference equations with a second order truncation error. The finite difference method is unique among other numerical techniques as it allows us to effectively control the rate of convergence via an initial approximation. Very few papers, however, have provided guidance for researchers as to customization of the Keller-box scheme to magnetohydrodynamic heat transfer problems. We therefore present a more detailed exposition here.

Essentially, 4 phases are central to the Keller Box scheme. These are

a. $\quad$ Reduction of the $N^{\text {th }}$ order partial differential equation system to $N$ first order equations.

b. Finite difference discretization.

c. Quasilinearization of non-linear Keller algebraic equations.

d. Block-tridiagonal elimination of linear Keller algebraic equations.

\section{Phase a: Reduction of the $N^{\text {th }}$ order partial differential equation system to $N$ first order equations}

Equations $(2.10)-(2.12)$ subject to the boundary conditions (2.13) are first written as a system of first-order equations. For this purpose, we reset Eqs (2.10) - (2.12) as a set of simultaneous equations by introducing the new variables $u, v, t$ and $p$

$$
\begin{aligned}
& f^{\prime}=u, \quad f^{\prime \prime}=v, \quad \theta^{\prime}=t, \quad \phi^{\prime}=p \\
& v^{\prime}+f v-(1+\xi \Lambda) u^{2}+\frac{\sin \xi}{\xi}(\theta+N \phi)-\left(M+\frac{1}{\mathrm{Da}}\right) u=\xi\left(u \frac{\partial u}{\partial \xi}-v \frac{\partial f}{\partial \xi}\right) \\
& \frac{1}{\operatorname{Pr}}\left(1+\frac{4}{3 F}\right) t^{\prime}+f t+\operatorname{Du} p^{\prime}=\xi\left(u \frac{\partial \theta}{\partial \xi}-t \frac{\partial f}{\partial \xi}\right) \\
& \frac{p^{\prime}}{\mathrm{Sc}}+f p+\operatorname{Sr} t^{\prime}=\xi\left(u \frac{\partial \phi}{\partial \xi}-p \frac{\partial f}{\partial \xi}\right)
\end{aligned}
$$


where primes denote differentiation with respect to $\eta$.

In terms of the dependent variables, the boundary conditions become

$$
\begin{aligned}
& \text { At } \quad \eta=0: \quad u=0, \quad f=f_{w}, \quad s=1, \quad g=1, \\
& \text { As } \quad \eta \rightarrow \infty: \quad u \rightarrow 0, \quad s \rightarrow 0, \quad g \rightarrow 0
\end{aligned}
$$

\section{Phase b: Finite difference discretization}

A two dimensional computational grid is imposed on the $\xi-\eta$ plane as sketched in Fig.2. The stepping process is defined by

$$
\begin{aligned}
& \eta_{0}=0, \quad \eta_{j}=\eta_{j-1}+h_{j}, \quad j=1,2, \ldots, J, \quad \eta_{J} \equiv \eta_{\infty}, \\
& \xi^{0}=0, \quad \xi^{n}=\xi^{n-1}+k_{n}, \quad n=1,2, \ldots, N
\end{aligned}
$$

where $k_{n}$ and $h_{j}$ denote the step distances in the $\xi$ and $\eta$ directions, respectively.

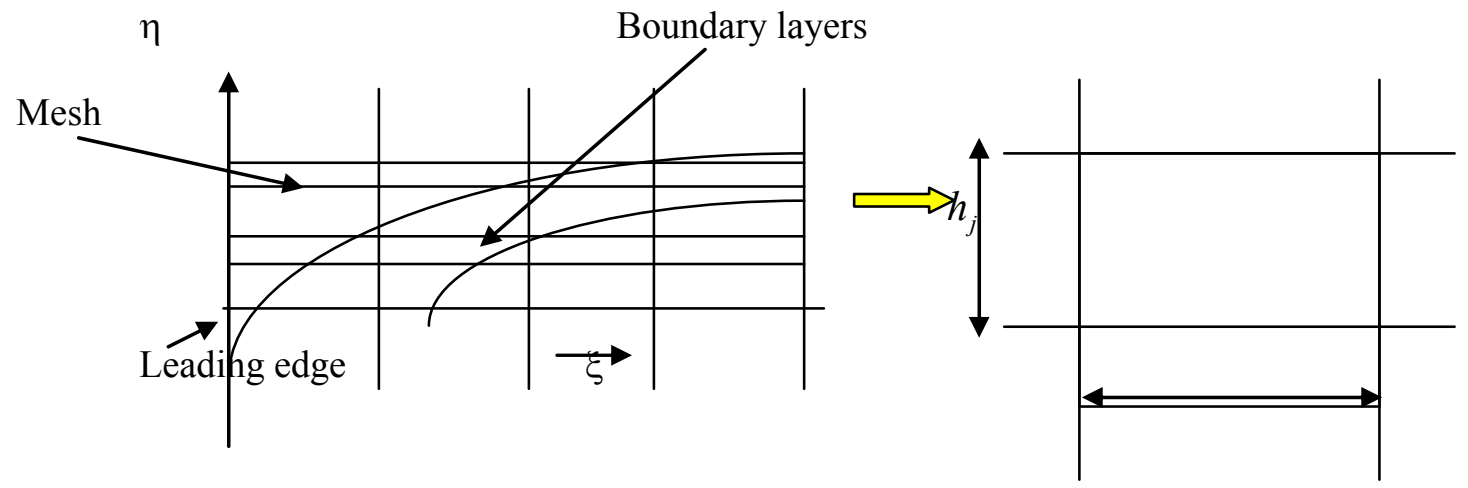

Fig.2. Grid meshing and a Keller box computational cell.

If $g_{j}^{n}$ denotes the value of any variable at $\left(\eta_{j}, \xi^{n}\right)$, then the variables and derivatives of Eqs (3.2)(3.4) at $\left(\eta_{j-1 / 2}, \xi^{n-1 / 2}\right)$ are replaced by

$$
\begin{aligned}
& g_{j-1 / 2}^{n-1 / 2}=\frac{1}{4}\left(g_{j}^{n}+g_{j-1}^{n}+g_{j}^{n-1}+g_{j-1}^{n-1}\right), \\
& \left(\frac{\partial g}{\partial \eta}\right)_{j-1 / 2}^{n-1 / 2}=\frac{1}{2 h_{j}}\left(g_{j}^{n}-g_{j-1}^{n}+g_{j}^{n-1}-g_{j-1}^{n-1}\right), \\
& \left(\frac{\partial g}{\partial \xi}\right)_{j-1 / 2}^{n-1 / 2}=\frac{1}{2 k^{n}}\left(g_{j}^{n}-g_{j-1}^{n}+g_{j}^{n-1}-g_{j-1}^{n-1}\right) .
\end{aligned}
$$

We now show the finite-difference approximation of Eqs (3.1) - (3.4) for the mid-point $\left(\eta_{j-1 / 2}, \xi^{n}\right)$, below 


$$
\begin{aligned}
& h_{j}^{-1}\left(f_{j}^{n}-f_{j-1}^{n}\right)=u_{j-1 / 2}^{n}, \\
& h_{j}^{-1}\left(u_{j}^{n}-u_{j-1}^{n}\right)=v_{j-1 / 2}^{n}, \\
& \left(v_{j}-v_{j-1}\right)+\frac{(1+\alpha) h_{j}}{4}\left[\left(f_{j}+f_{j-1}\right)\left(v_{j}+v_{j-1}\right)\right]-\frac{h_{j}}{4}(1+\alpha+\xi \Lambda)\left(u_{j}+u_{j-1}\right)^{2}+ \\
& -\frac{h_{j}}{2}\left(M+\frac{1}{D a}\right)\left(u_{j}+u_{j-1}\right)+\frac{\alpha \varepsilon^{-2} h_{j}}{2} v_{j-1 / 2}^{n-1}\left(f_{j}+f_{j-1}\right)+ \\
& -\frac{\alpha \varepsilon^{-2} h_{j}}{2} f_{j-1 / 2}^{n-1}\left(v_{j}+v_{j-1}\right)+\frac{B h_{j}}{2}\left[\theta_{j}+\theta_{j-1}+N\left(\phi_{j}+\phi_{j-1}\right)\right]=\left[R_{1}\right]_{j-1 / 2}^{n-1}, \\
& h_{j}^{-1}\left(\theta_{j}^{n}-\theta_{j-1}^{n}\right)=t_{j-1 / 2}^{n}, \\
& \frac{1}{\operatorname{Pr}}\left(1+\frac{4}{3 F}\right)\left(t_{j}-t_{j-1}\right)+\frac{(1+\alpha) h_{j}}{4}\left[\left(f_{j}+f_{j-1}\right)\left(t_{j}+t_{j-1}\right)\right]-\frac{\alpha h_{j}}{4}\left[\left(u_{j}+u_{j-1}\right)\left(\theta_{j}+\theta_{j-1}\right)\right]+ \\
& +\frac{\alpha h_{j}}{2} \theta_{j-1 / 2}^{n-1}\left(u_{j}+u_{j-1}\right)-\frac{\alpha h_{j}}{2} u_{j-1 / 2}^{n-1}\left(\theta_{j}+\theta_{j-1}\right)-\frac{\alpha h_{j}}{2} f_{j-1 / 2}^{n-1}\left(t_{j}+t_{j-1}\right)+ \\
& +\frac{\alpha h_{j}}{2} t_{j-1 / 2}^{n-1}\left(f_{j}+f_{j-1}\right)+D u\left(p_{j}-p_{j-1}\right)=\left[R_{2}\right]_{j-1 / 2}^{n-1} \\
& h_{j}^{-1}\left(\phi_{j}^{n}-\phi_{j-1}^{n}\right)=p_{j-1 / 2}^{n}, \\
& \frac{1}{\mathrm{Sc}}\left(p_{j}-p_{j-1}\right)+\frac{(1+\alpha) h_{j}}{4}\left[\left(f_{j}+f_{j-1}\right)\left(p_{j}+p_{j-1}\right)\right]-\frac{\alpha h_{j}}{4}\left[\left(u_{j}+u_{j-1}\right)\left(\phi_{j}+\phi_{j-1}\right)\right]+ \\
& +\frac{\alpha h_{j}}{2} \phi_{j-1 / 2}^{n-1}\left(u_{j}+u_{j-1}\right)-\frac{\alpha h_{j}}{2} u_{j-1 / 2}^{n-1}\left(\phi_{j}+\phi_{j-1}\right)-\frac{\alpha h_{j}}{2} f_{j-1 / 2}^{n-1}\left(p_{j}+p_{j-1}\right)+ \\
& +\frac{\alpha h_{j}}{2} p_{j-1 / 2}^{n-1}\left(f_{j}+f_{j-1}\right)+\operatorname{Sr}\left(t_{j}-t_{j-1}\right)=\left[R_{3}\right]_{j-1 / 2}^{n-1}
\end{aligned}
$$

where we have used the abbreviations

$$
\begin{aligned}
& \alpha=\frac{\xi^{n-1 / 2}}{k_{n}}, \quad B=\frac{\sin \left(\xi^{n-1 / 2}\right)}{\xi^{n-1 / 2}}, \\
& {\left[R_{1}\right]_{j-1 / 2}^{n-1}=-h_{j}\left[\left(\frac{v_{j}-v_{j-1}}{h_{j}}\right)+(1-\alpha)\left(f_{j-1 / 2} v_{j-1 / 2}\right)+(\alpha-1-\xi \Lambda)\left(u_{j-1 / 2}\right)^{2}+\right.} \\
& \left.+B\left(\theta_{j-1 / 2}+N \phi_{j-1 / 2}\right)-\left(M+\frac{1}{\mathrm{Da}}\right) u_{j-1 / 2}\right],
\end{aligned}
$$




$$
\begin{aligned}
& {\left[R_{2}\right]_{j-1 / 2}^{n-1}=} \\
& =-h_{j}\left[\frac{1}{\operatorname{Pr}}\left(1+\frac{4}{3 F}\right)\left(\frac{t_{j}-t_{j-1}}{h_{j}}\right)+(1-\alpha)\left(f_{j-1 / 2} t_{j-1 / 2}\right)+\alpha\left(u_{j-1 / 2} \theta_{j-1 / 2}\right)+\operatorname{Du}\left(\frac{p_{j}-p_{j-1}}{h_{j}}\right)\right], \\
& {\left[R_{3}\right]_{j-1 / 2}^{n-1}=} \\
& =-h_{j}\left[\frac{1}{\operatorname{Sc}}\left(\frac{p_{j}-p_{j-1}}{h_{j}}\right)+(1-\alpha)\left(f_{j-1 / 2} p_{j-1 / 2}\right)+\alpha\left(u_{j-1 / 2} \phi_{j-1 / 2}\right)+\operatorname{Sr}\left(\frac{t_{j}-t_{j-1}}{h_{j}}\right)\right] .
\end{aligned}
$$

The boundary conditions are

$$
f_{0}^{n}=u_{0}^{n}=0, \quad \theta_{0}^{n}=1, \quad \phi_{0}^{n}=1, \quad u_{J}^{n}=0, \quad \theta_{J}^{n}=0, \quad \phi_{J}^{n}=0 .
$$

\section{Phase c: Quasi-linearization of non-linear Keller algebraic equations}

If we assume $f_{j}^{n-1}, u_{j}^{n-1}, v_{j}^{n-1}, \theta_{j}^{n-1}, t_{j}^{n-1}, \phi_{j}^{n-1}, p_{j}^{n-1}$ to be known for $0 \leq j \leq J$, Eqs (3.8a) - (2.8g) are a system of 7J+7 equations for the solution of 7 $\mathbf{J}+7$ unknowns $f_{j}^{n}, u_{j}^{n}, v_{j}^{n}, \phi_{j}^{n}, p_{j}^{n}, \theta_{j}^{n}, t_{j}^{n} j=0,1,2 \ldots, J$. This nonlinear system of algebraic equations is linearized by means of Newton's method as explained in Vasu et al. [31].

\section{Phase d: Block-tridiagonal elimination of linear Keller algebraic equations}

The linear system (3.8) can now be solved by the block-elimination method. The linearized difference equations of the system have a block-tridiagonal structure. Commonly, the block-tridiagonal structure consists of variables or constants, but here, an interesting feature can be observed, that is, for the Keller-box method, it consists of block matrices. Following the procedure, as explained in Vasu et al. [31], the complete linearized system is formulated as block matrix system, where each element in the coefficient matrix is a matrix itself. The numerical results are affected by the number of mesh points in both directions. After some trials in the $\eta$ direction a larger number of mesh points are selected, whereas in the $\xi$ direction significantly less mesh points are utilized. $\eta_{\max }$ has been set at 40 and this defines an adequately large value at which the prescribed boundary conditions are satisfied. $\xi_{\max }$ is set at 3.0 for this flow domain. Accurate results are produced by performing a mesh sensitivity analysis (shown in Tab.2) and convergence criteria. Further details of the solution procedure are documented in for example Vasu et al. [31], and omitted here to save of space.

\section{Calculations of system performance}

The computations were carried out on WIPRO Computer of Processor: Intel I Core(TM) i5 CPU 760 (a) 2.80Ghz, 2.79Ghz with internal memory (RAM): $4.00 \mathrm{~GB}$ running by Windows 7 Professional 64-bit Operating System. In order to measure the performance of calculation, we calculated the computation time of the program in MATLAB using CPUTIME function, the TIMEIT or TIC and TOC functions and CLOCK function, which enable to time how long the code takes to run. Table 1 shows the current calculation time in seconds for different grid points, time taken for running the program increases together with an increase in the number of grid points. This agrees the physical relevance in increasing the complexity and nonlinearity in the calculation. 
Table 1. Calculation of computation time to measure the performance of MATLAB program when $\mathrm{Da}=0.1$, $\Lambda=0.1, \operatorname{Pr}=0.71, N=1, f_{w}=0.5, \mathrm{Sc}=0.25, F=0.5, \mathrm{Sr}=0.25, \mathrm{Du}=0.2$.

\begin{tabular}{|c|c|c|c|c|}
\hline \multirow{2}{*}{ S No } & \multirow{2}{*}{ Grid or mesh } & \multicolumn{3}{|c|}{ Calculation of computation time } \\
\cline { 3 - 5 } & & TIC TOC & CPU Time & Clock \\
\hline 1 & $11 \times 1501$ & 14.8875 & 14.8669 & 14.889 \\
\hline 2 & $21 \times 1501$ & 34.5117 & 34.4138 & 34.513 \\
\hline 3 & $31 \times 1501$ & 62.3754 & 62.1352 & 62.378 \\
\hline 4 & $31 \times 2001$ & 102.404 & 102.227 & 102.41 \\
\hline 5 & $31 \times 2501$ & 165.049 & 164.721 & 165.058 \\
\hline
\end{tabular}

Generally, for CPU-intensive calculations that run on Microsoft ${ }^{\circledR}$ Windows ${ }^{\circledR}$ machines, the elapsed time from CPUTIME and from TIC and TOC are close in value, ignoring any first-time costs. There are cases, however, that show a significant difference between these functions. Like TIC and TOC, TIMEIT provides more reliable results than CPUTIME. However, the TIMEIT function also considers first-time costs.

\section{Grid sensitivity analysis}

Several different grid distributions have been tested to ensure that the calculated results are grid independent. Table 2 shows the comparison of the skin friction $\left(\xi f^{\prime \prime}(\xi, 0)\right)$, Nusselt $\left(-\theta^{\prime}(\xi, 0)\right)$ and Sherwood $\left(-\phi^{\prime}(\xi, 0)\right)$ coefficients values for the different grid distributions. A uniform grid distribution has been used to discretize the computational domain. We noticed that increasing the grid numbers in the computation domain does not change significantly the Skin friction, Nusselt and Sherwood coefficients values. Therefore, the grid which consists of 31 and 2001 nodes in the horizontal and vertical directions, respectively has been selected for the present calculations. It is shown that $\left(\xi f^{\prime \prime}(\xi, 0)\right),\left(-\theta^{\prime}(\xi, 0)\right)$ and $\left(-\phi^{\prime}(\xi, 0)\right)$ values are independent of the number of grid points when the thermophysical values $\mathrm{Da}=0.1, \Lambda=0.1, \operatorname{Pr}=0.71$, $N=1, f_{w}=0.5, \mathrm{Sc}=0.25, F=0.5, \mathrm{Sr}=0.25, \mathrm{Du}=0.2$.

Table 2. Grid independence analysis when $\mathrm{Da}=0.1, \Lambda=0.1, \operatorname{Pr}=0.71, N=1, f_{w}=0.5, \mathrm{Sc}=0.25, F=0.5$, $\mathrm{Sr}=0.25, \mathrm{Du}=0.2$.

\begin{tabular}{|c|c|c|c|c|}
\hline S. No & Mesh & $\xi f^{\prime \prime}(\xi, 0)$ & $-\theta^{\prime}(\xi, 0)$ & $-\phi^{\prime}(\xi, 0)$ \\
\hline 1 & $11 \times 501$ & 0.504095158573570 & 0.176151036859258 & 0.202775932535556 \\
\hline 2 & $11 \times 1001$ & 0.507258446573282 & 0.153562294768314 & 0.187507133142110 \\
\hline 3 & $11 \times 1501$ & 0.507469223969663 & 0.151682025580220 & 0.186866447056417 \\
\hline 4 & $11 \times 2001$ & 0.507487404379979 & 0.151496063763370 & 0.186834971147045 \\
\hline 5 & $11 \times 2501$ & 0.507489134570660 & 0.151476917086506 & 0.186833411101278 \\
\hline 6 & $21 \times 501$ & 0.536397017514021 & 0.145057355152699 & 0.162812195485454 \\
\hline 7 & $21 \times 1001$ & 0.541279510767156 & 0.113509758467140 & 0.139345138517288 \\
\hline 8 & $21 \times 1501$ & 0.541659584227665 & 0.110381422017085 & 0.138193105087817 \\
\hline 9 & $21 \times 2001$ & 0.541700758420850 & 0.109977228625472 & 0.138133585394828 \\
\hline 10 & $21 \times 2501$ & 0.541706184576448 & 0.109917107740069 & 0.138132533358192 \\
\hline 11 & $31 \times 501$ & 0.0562813476618773 & 0.108559160200178 & 0.111428411003198 \\
\hline 12 & $31 \times 1001$ & 0.0577083727913430 & 0.0342643034319676 & 0.0399389574362061 \\
\hline 13 & $31 \times 1501$ & 0.0578190152471138 & 0.0265703137920884 & 0.0361104361629599 \\
\hline $\mathbf{1 4}$ & $31 \times 2001$ & $\mathbf{0 . 0 5 7 8 2 7 3 1 9 9 3 6 8 2 8 2}$ & $\mathbf{0 . 0 2 5 8 0 6 9 2 8 1 9 1 0 0 7 1}$ & $\mathbf{0 . 0 3 5 9 3 0 2 1 2 8 0 0 3 1 4 9}$ \\
\hline 15 & $31 \times 2501$ & 0.0578284624512443 & 0.0256815693267795 & 0.0359206028650375 \\
\hline
\end{tabular}




\section{Results and discussion}

A representative set of numerical results is presented graphically to illustrate the influence of the hydromagnetic parameter $(M)$, Darcy number $(\mathrm{Da})$, Prandtl number $(\mathrm{Pr})$, tangential coordinate $(\xi)$, radiation parameter $(F)$ and Schmidt number $(\mathrm{Sc})$ on velocity, temperature, concentration, shear stress, local Nusselt number and Sherwood number profiles. In all cases we have assumed the following default values (unless otherwise stated) for the parameters: $\operatorname{Pr}=0.71$ (air), $\Lambda=0.1$ (weak second order Forchheimer drag), $F=0.5$, $M=1.0$ (equivalent hydromagnetic and viscous forces), $\mathrm{Da}=0.1$ (very high permeability of regime), $f_{w}=0.5$.

In order to verify the accuracy of our present method, we have compared our results with those of Merkin [32] and Yih [27]. Table 3 shows the comparisons of the values of $-\theta^{\prime}(\xi, 0)$. It is also observed from Tab. 3 that the temperature gradient is clearly decreased with increasing the distance from the leading edge, i.e. along the cylinder surface from the leading edge.

Table 3. Values of the local heat transfer coefficient $(\mathrm{Nu})$ for various values of $\xi$ with $\mathrm{Da} \rightarrow \infty, \Lambda=0$, $\operatorname{Pr}=1, N=0, f_{w}=0, \mathrm{Sc}=0, F \rightarrow \infty, \mathrm{Sr}=\mathrm{Du}=0$.

\begin{tabular}{|c|c|c|c|}
\hline \multirow{2}{*}{} & \multicolumn{3}{|c|}{$-\theta^{\prime}(\xi, 0)$} \\
\cline { 2 - 4 } & Merkin [32] & Yih [27] & Present results \\
\hline 0.0 & 0.4212 & 0.4214 & 0.4214 \\
0.4 & 0.4182 & 0.4184 & 0.4185 \\
0.8 & 0.4093 & 0.4096 & 0.4097 \\
1.2 & 0.3942 & 0.3950 & 0.3952 \\
1.6 & 0.3727 & 0.3740 & 0.3741 \\
2.0 & 0.3443 & 0.3457 & 0.3460 \\
2.4 & 0.3073 & 0.3086 & 0.3087 \\
2.8 & 0.2581 & 0.2595 & 0.2597 \\
$\pi$ & 0.1963 & 0.1962 & 0.1964 \\
\hline
\end{tabular}

Figures $3 \mathrm{a}-3 \mathrm{c}$ show the effect of the Darcy number (Da) on dimensionless velocity $\left(f^{\prime}\right)$, temperature $(\theta)$ and concentration $(\phi)$ with transformed radial coordinate $(\xi)$ at a location close to the lower stagnation point $(\xi=0.1) . \mathrm{Da}=\frac{K \sqrt{\mathrm{Gr}}}{a^{2}}$, for a fixed value of the cylinder radius $a$, and the free convection parameter Gr (Grashof number) is directly proportional to permeability $K$, of the porous regime. In the momentum conservation Eq.(2.10), the Darcian drag term, $-\left(\frac{1}{\mathrm{Da}}\right) f^{\prime}$, is inversely proportional to Da. Increasing $\mathrm{Da}$ increases the porous medium permeability and simultaneously decreases the Darcian impedance since progressively less solid fibers are present in the regime. The flow is therefore accelerated for higher Da values causing an increase in the velocity $\left(f^{\prime}\right)$ as shown in Fig.3a. Maximum effect of rising the Darcy number is observed at an intermediate distance from the cylinder surface around $\eta \sim 1$. It is noted that all the profiles correspond to a location some distance from the lower stagnation point on the cylinder at $\xi=0.5$. Conversely, the temperature $\theta$ depicted in Fig. $3 \mathrm{~b}$ is opposed by increasing the Darcy number. The presence of fewer solid fibers in the regime with increasing $\mathrm{Da}$, inhibits the thermal conduction in the medium which reduces distribution of thermal energy. The regime is therefore cooled when more fluid is present and $\theta$ values in the thermal boundary layer are decreased. Profiles for both velocity and temperature are smoothly decay to the free stream indicating that excellent convergence (and stability) is obtained with 
the numerical method. Velocity boundary layer thickness will be increased with a rise in Da and thermal boundary layer thickness is reduced. Concentration values are also reduced with increasing the Darcy number (Da) as seen in Fig.3c.

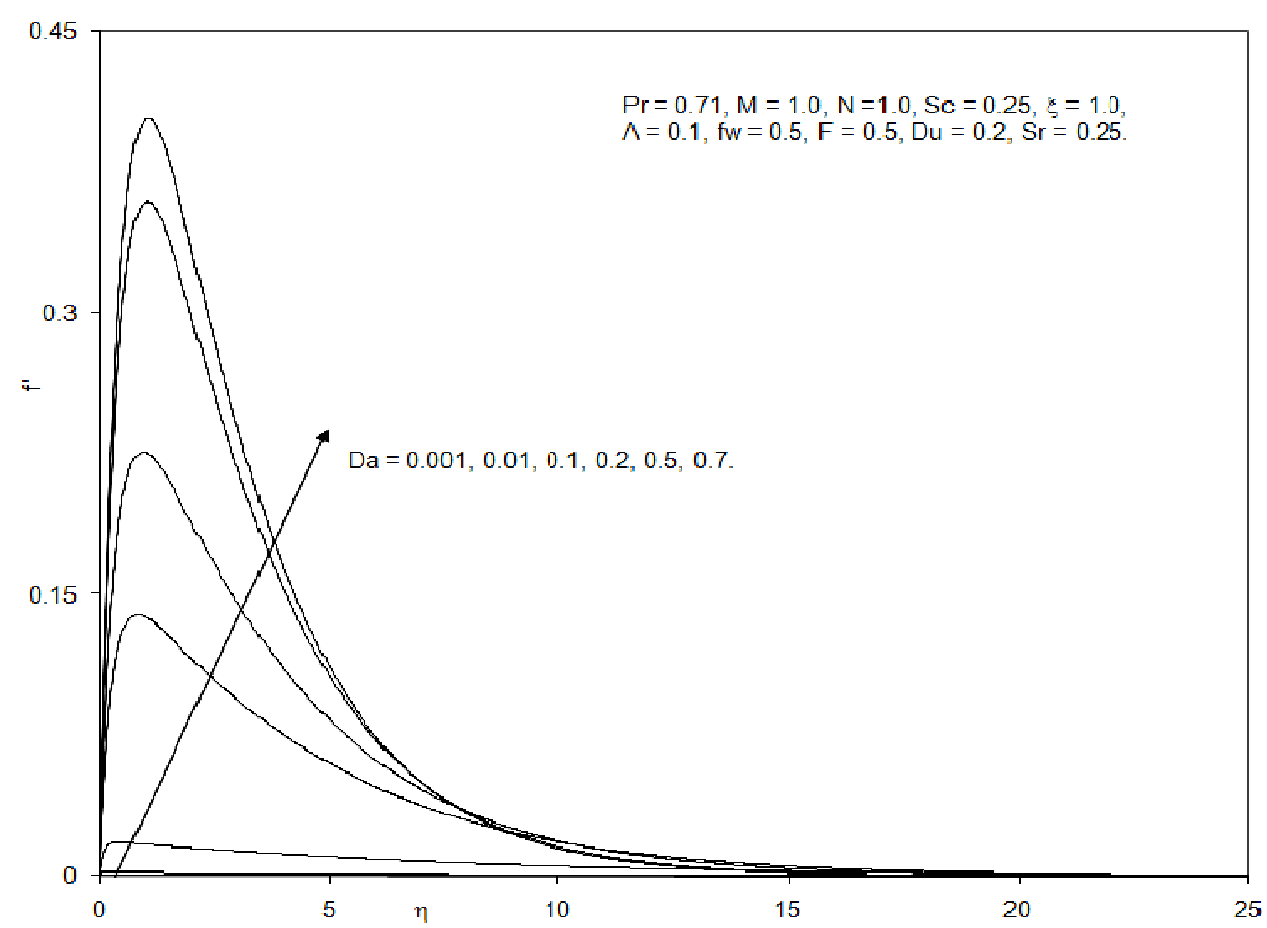

Fig.3a. Effect of the Da on the velocity profiles.

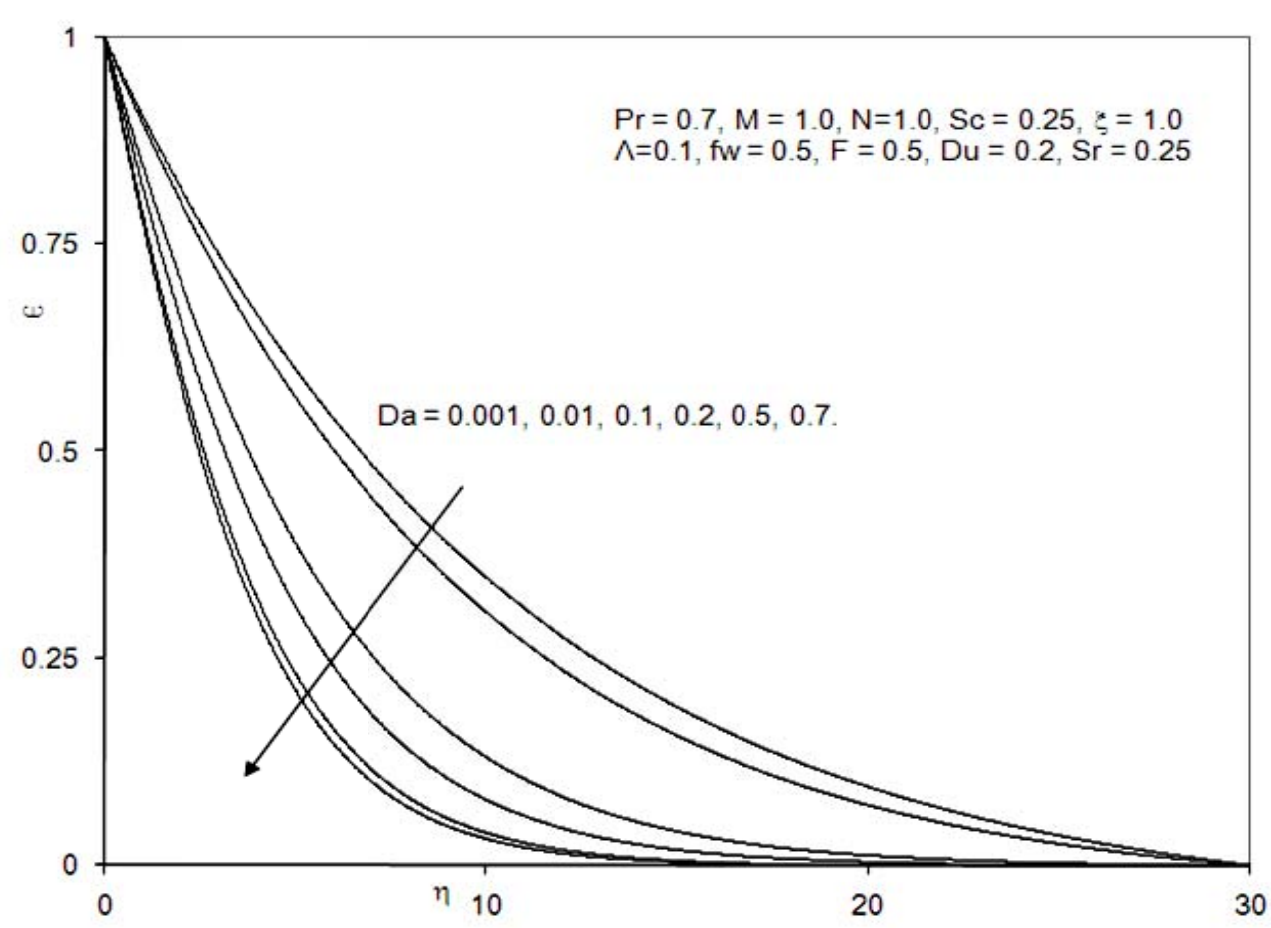

Fig.3b. Effect of the Da on the temperature profiles. 


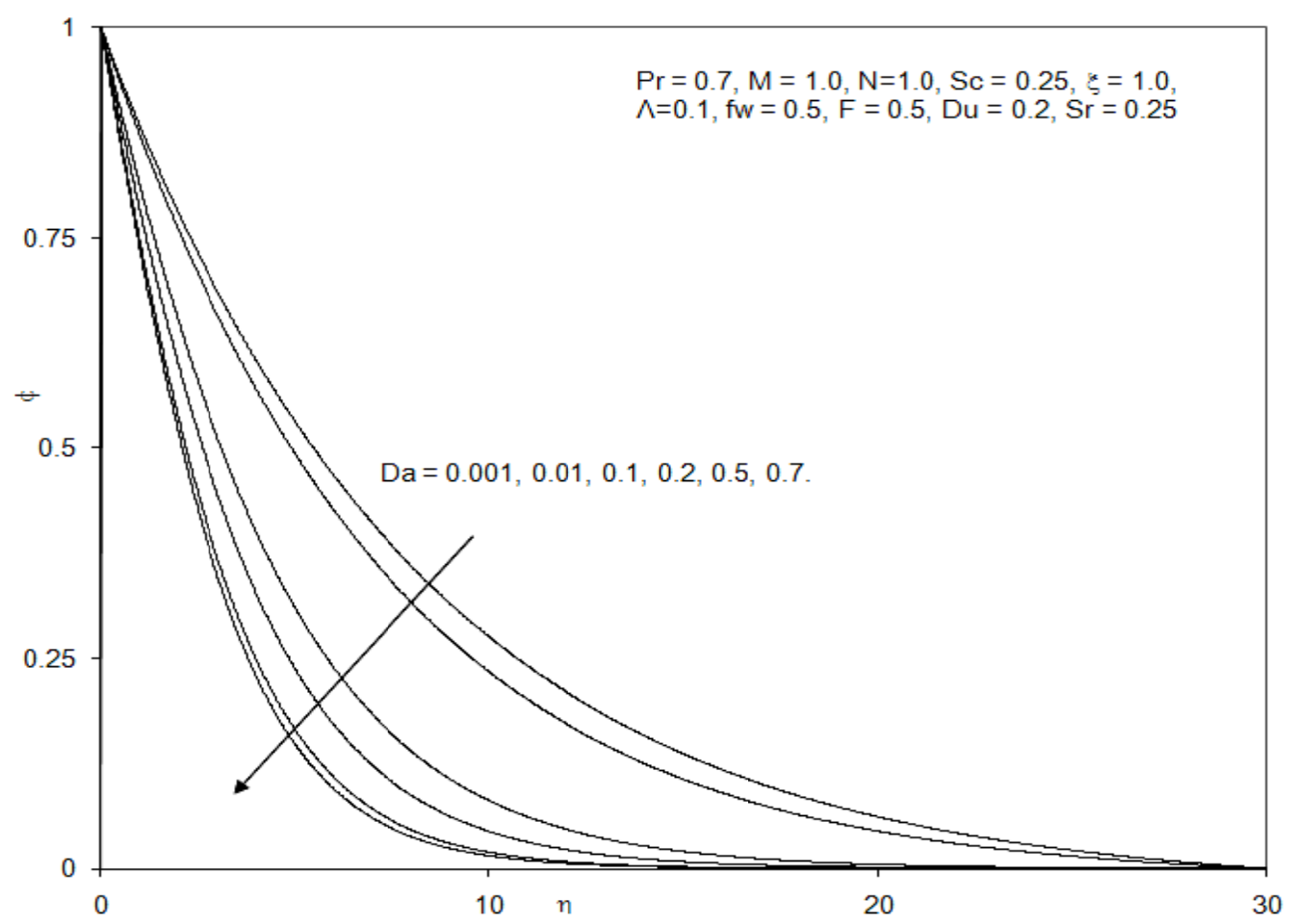

Fig.3c. Effect of the Da on the concentration profiles.

For different values of the radiation parameter $F$, the velocity, temperature and concentration profiles are plotted in Figs $4 \mathrm{a}-4 \mathrm{c} . F=\frac{k k_{e}}{4 \sigma^{*} T_{\infty}^{3}}$ and this defines the ratio of thermal conduction contribution relative to the thermal radiation. Radiative heat transfer dominates in the boundary layer regime when $F \rightarrow 0$. For finite values of $F$ there will be a simultaneous presence of thermal conduction and radiative transfer contribution. For $F=1$ both modes will contribute equally. For $F \rightarrow \infty$, in Eq.(2.11), the term $4 / 3 F \rightarrow 0$ and the energy conservation equation reduces to the convectional steady conduction-convection equation with dissipation, i.e.

$$
\frac{1}{\operatorname{Pr}} \theta^{\prime \prime}+f \theta^{\prime}=\xi\left(f^{\prime} \frac{\partial \theta}{\partial \xi}-\theta^{\prime} \frac{\partial f}{\partial \xi}\right)
$$

An increase in $F$ from 0.1 (total thermal radiation dominance) through 3.0, 5.0 to 100 , causes a significant decrease in velocity with distance into the boundary layer, i.e. it decelerates the flow. Velocities in all cases ascend from the cylinder surface, peak close to the wall and then decay smoothly to zero in the free stream. Thermal radiation flux therefore has a de-stabilizing effect on the flow regime. This is important in polymeric and other industrial flow processes since it shows that the presence of thermal radiation while decreasing temperature, will affect flow control from the cylinder surface into the boundary layer regime. As expected, temperature values are also significantly reduced with an increase in $F$ as there is a progressive decrease in thermal radiation contribution accompanying this. All profiles monotonically decay from the wall to the free stream. The maximum reduction in temperature is witnessed relatively close to the cylinder surface since thermal conduction effects will be prominent closer to the cylinder surface, rather than further into the free stream. Concentration is conversely boosted with an increase in $F$, i.e. decrease in thermal radiation contribution. The parameter $F$ does not arise in the species conservation Eq.(2.12) and therefore the 
concentration field is indirectly influenced by $F$ via the coupling of the energy Eq.(2.11) with the momentum Eq.(2.10), the latter also being coupled with the conservative acceleration terms in the species Eq.(2.12).

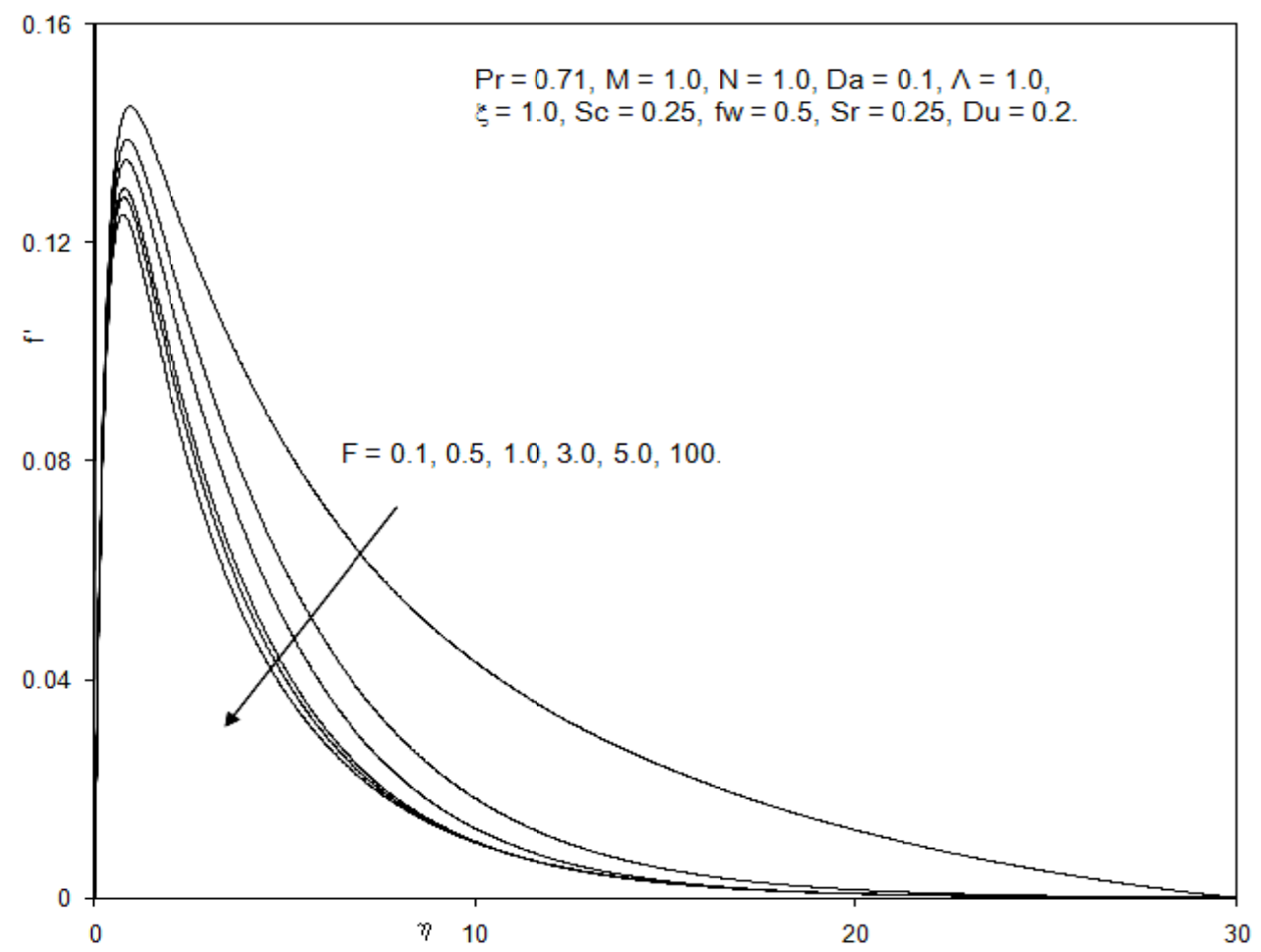

Fig.4a. Effect of the $F$ on the velocity profiles.

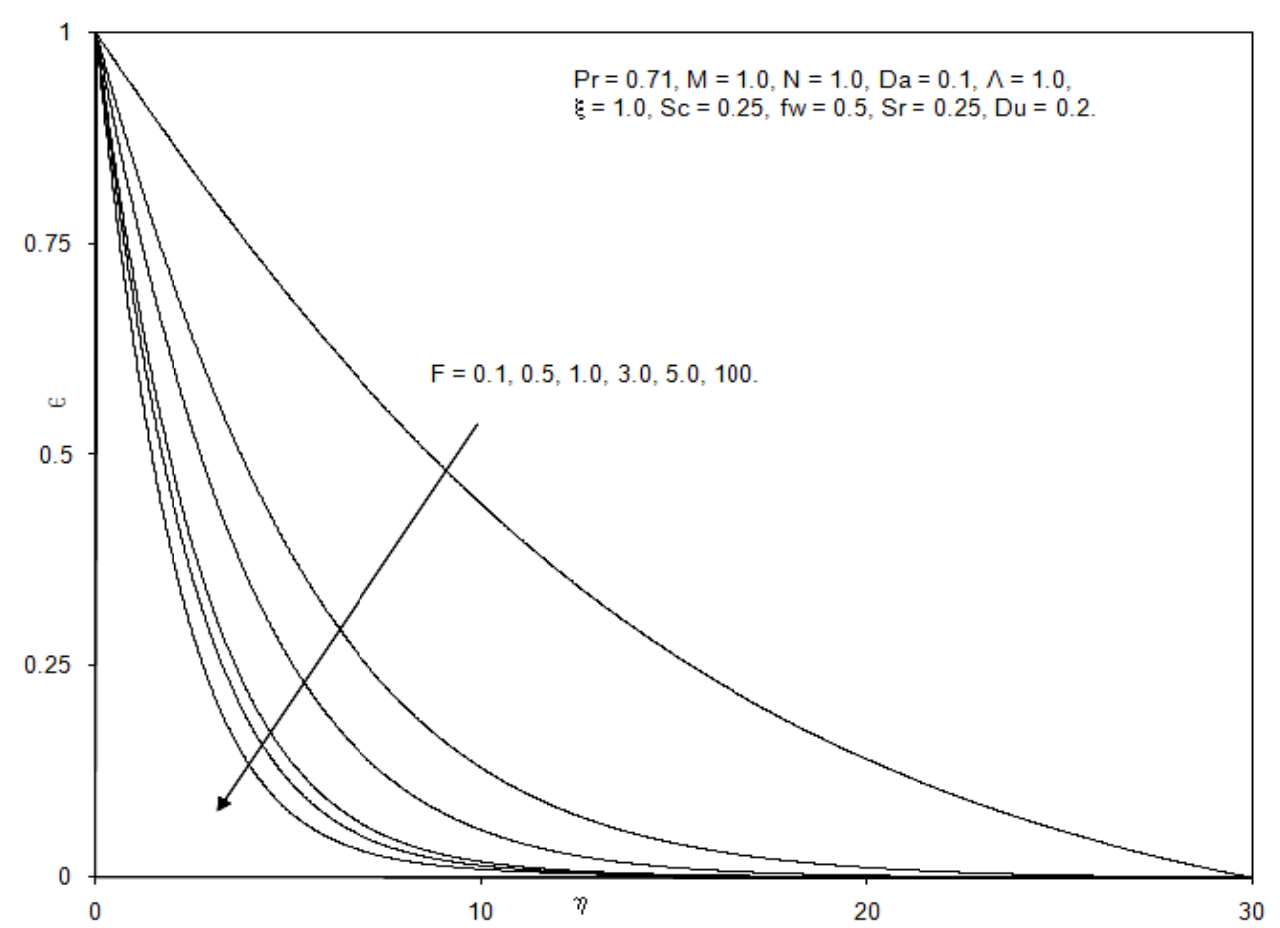

Fig.4b. Effect of the $F$ on the temperature profiles. 


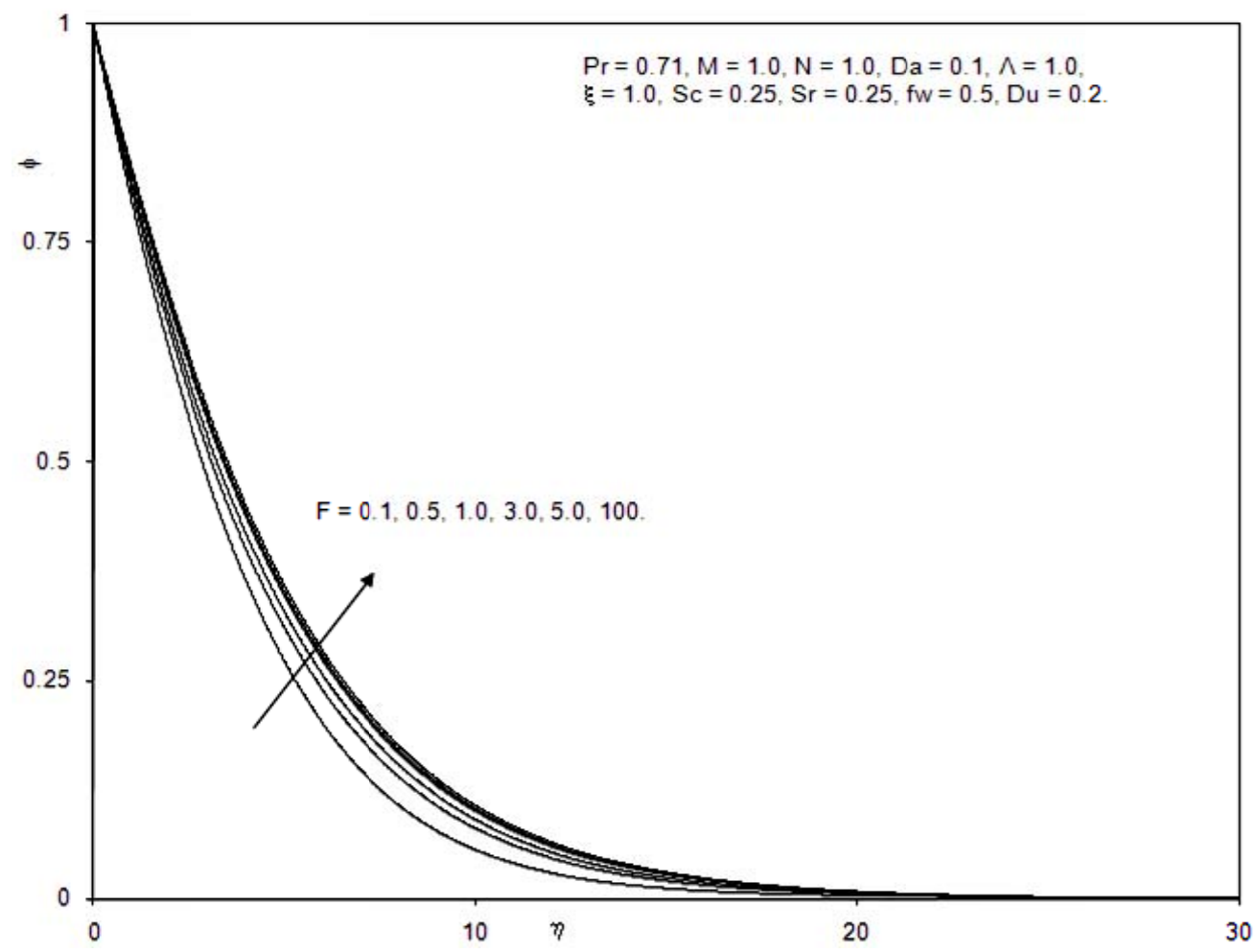

Fig.4c. Effect of the $F$ on the concentration profiles.

Figures $5 \mathrm{a}-5 \mathrm{c}$ illustrates the effect of the Prandtl number, $\operatorname{Pr}$ on velocity, temperature, and concentration. Increasing Pr clearly reduces strongly velocity, (Fig.5a) both in the near-wall regime and the far-field regime of the boundary layer. Velocity is therefore maximized when $\operatorname{Pr}=0.71$ (minimum) and minimized for the largest value of $\operatorname{Pr}(7.0)$. Pr defines the ratio of momentum diffusivity $(v)$ to thermal diffusivity. $\operatorname{Pr}<1$ physically corresponds to cases where heat diffuses faster than momentum. $\operatorname{Pr}=0.7$ is representative of water-based solvents and $\operatorname{Pr}>>1$, e.g. 5.4 corresponds to lubricating oils, etc. With increasing Pr from 0.7 through 1.0, 2.0, 4.0, 5.4 to 7.0, temperature as shown in Fig.5b, is markedly reduced throughout the boundary layer. The descent is increasingly sharper near the plate surface for higher $\operatorname{Pr}$ values. With lower Pr values a more gradual (monotonic) decay is witnessed. Smaller Pr values in this case cause a thinner thermal boundary layer thickness and more uniform temperature distributions across the boundary layer. Smaller Pr fluids possess higher thermal conductivities so that heat can diffuse away from the plate surface (wall) faster than for higher Pr fluids (thicker boundary layers). Our computations show that a rise in Pr depresses the temperature function, a result consistent with numerous other studies on coupled heat and mass transfer as indicated by Gebhart and Pera [33]. For the case of $\operatorname{Pr}=1$, thermal and velocity boundary layer thicknesses are equal. Conversely the concentration values (Fig.5c) are slightly increased with a rise in Pr from 0.71 through intermediate values to 7.0. However, with progression of time the concentration is found to be decreased in the boundary layer regime. 


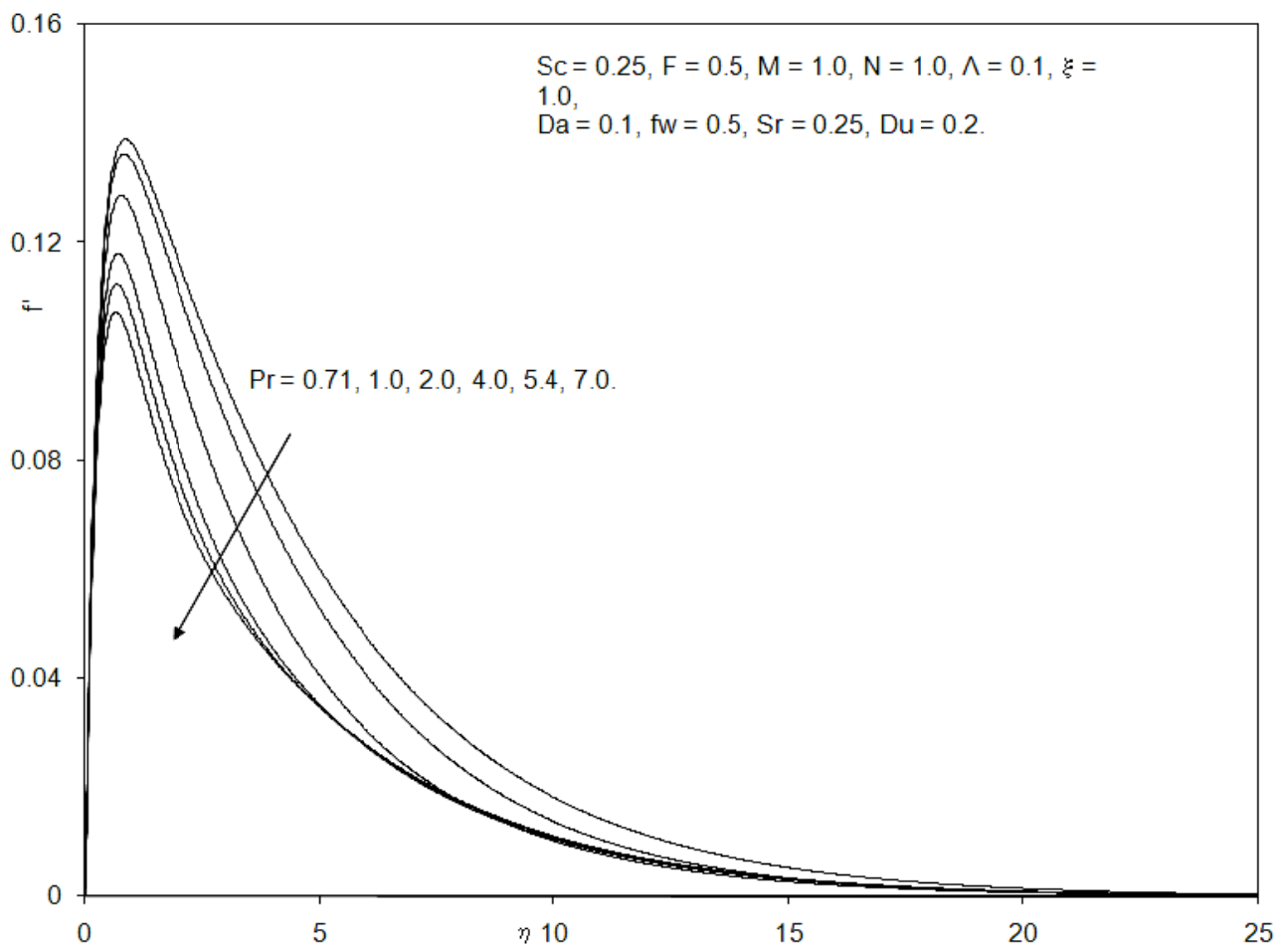

Fig.5a. Effect of the Pr on the velocity profiles.

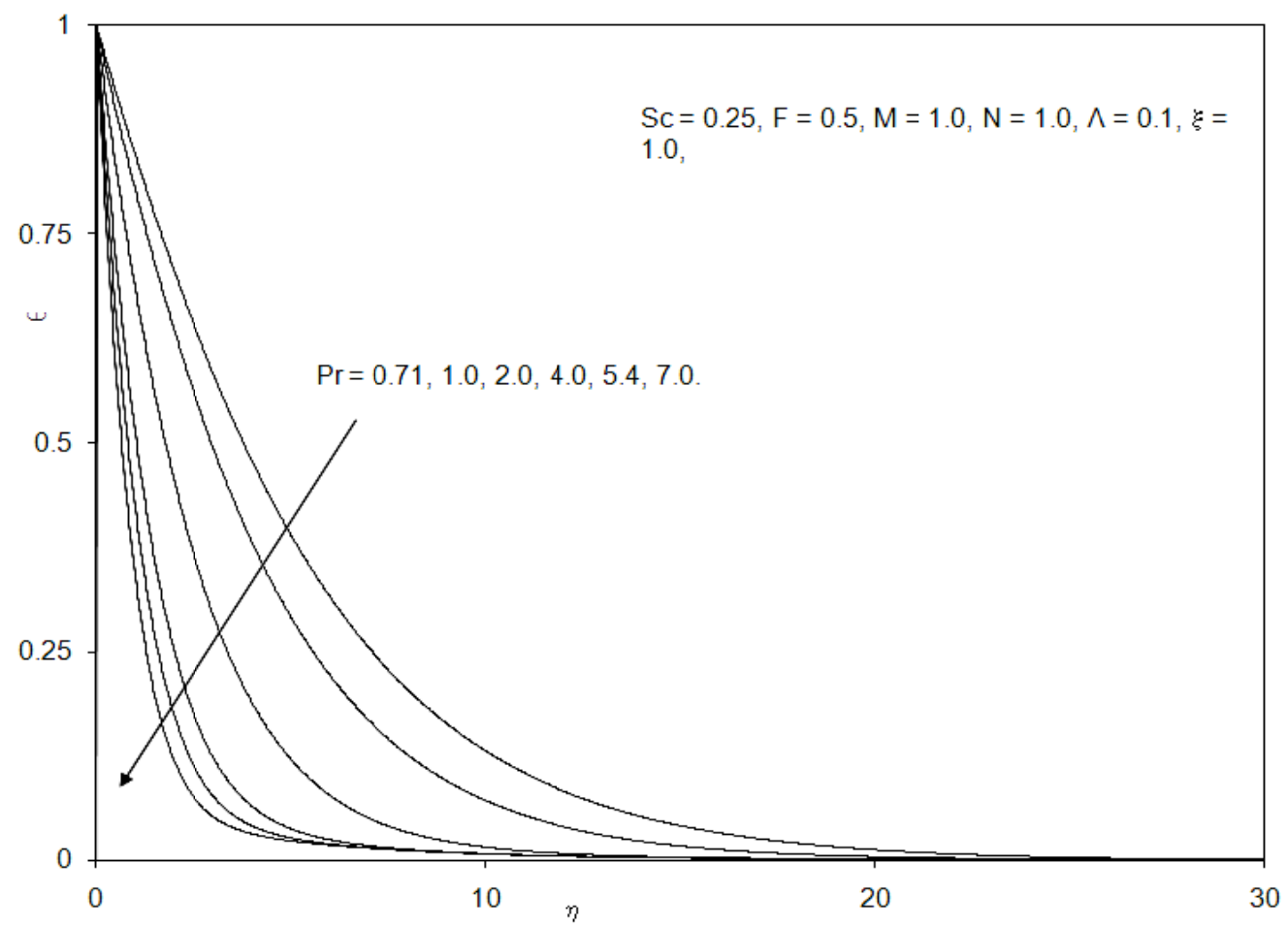

Fig.5b. Effect of the Pr on the temperature profiles. 


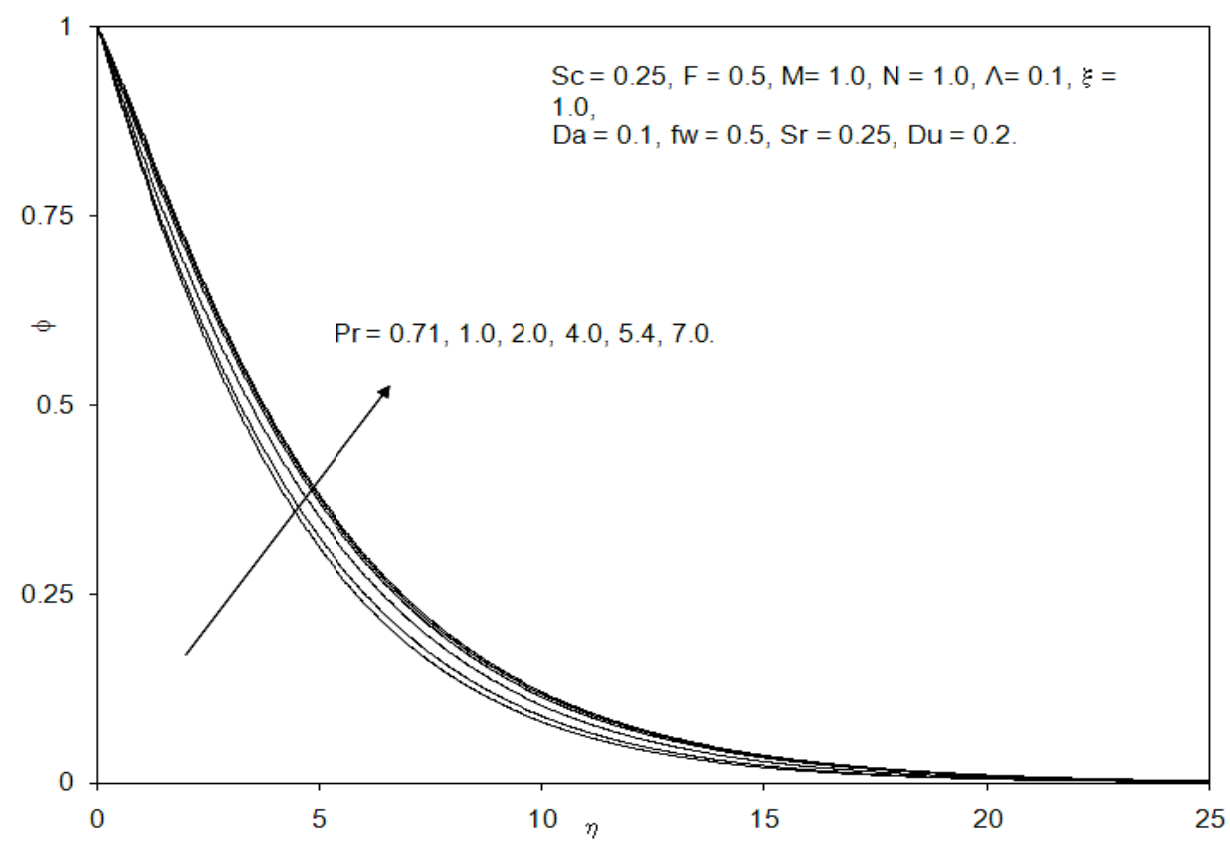

Fig.5c. Effect of the Pr on the concentration profiles.

Figures $6 a-6 c$ illustrate the linear velocity, temperature and concentration profile response to variation in the Schmidt number, Sc. With an increase in Sc from 0.25 through 0.6, 0.78, 0.94 to 2.0, the velocity is strongly reduced in the regime. The Schmidt number signifies the relative effect of momentum diffusion to species diffusion. For $\mathrm{Sc}<1$, species diffusivity dominates and vice versa for $\mathrm{Sc}>1$. $\mathrm{Sc}=0.25$ corresponds to approximately hydrogen at 25 Celsius and 1 atmosphere pressure. Larger values may simulate well methanol diffusing in air $(\mathrm{Sc}=1.0)$ and ethylbenzene in air $(\mathrm{Sc}=2.0)$, as indicated by Gebhart and Pera [33]. Our numerical calculations are executed for $\operatorname{Pr}=0.7$, so that $\operatorname{Pr} \neq \mathrm{Sc}$, and physically this implies that the thermal and species diffusion regions are of different extents. Temperature $\theta$ and species concentration $\phi$ both continuously increase with a rise in the Schmidt number. The presence of buoyancy-assisted flow $(N>0)$ aids in boosting temperatures also.

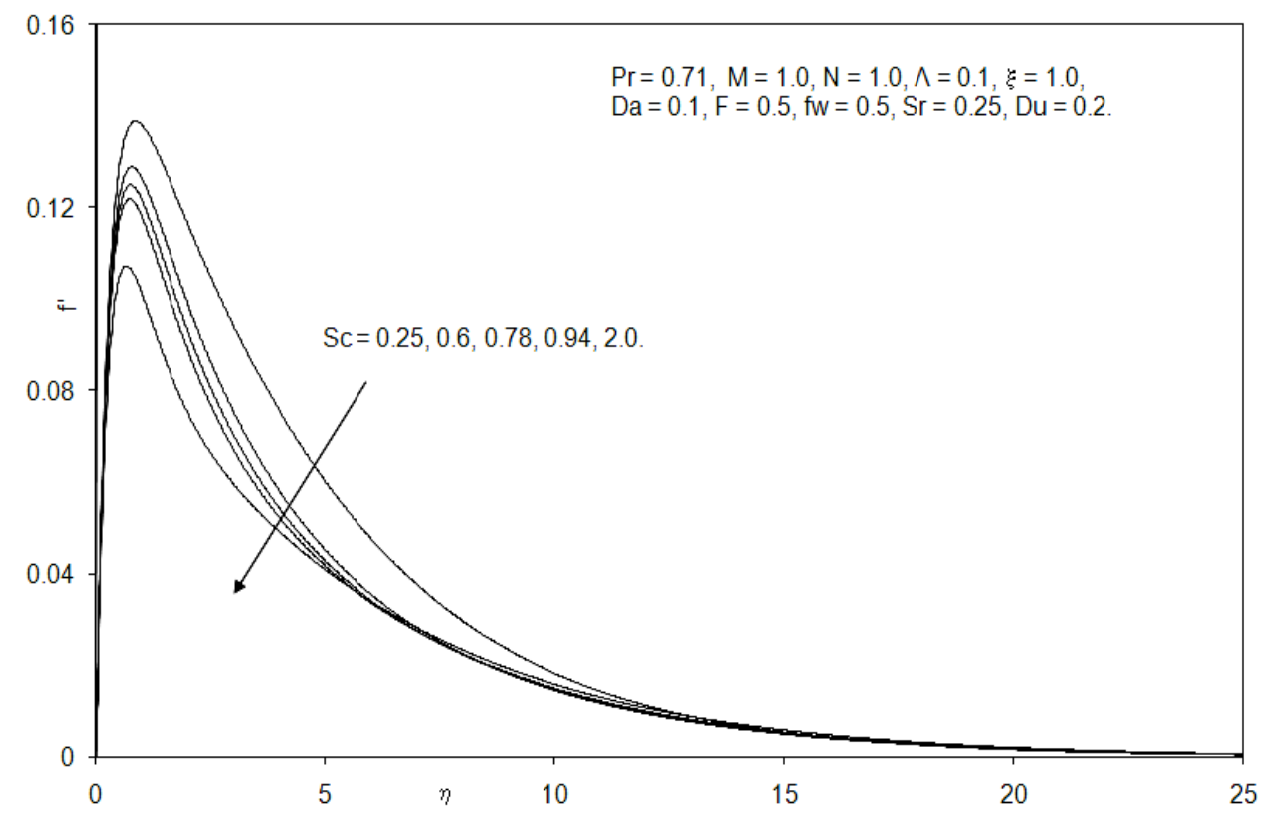

Fig.6a. Effect of the Sc on the velocity profiles. 


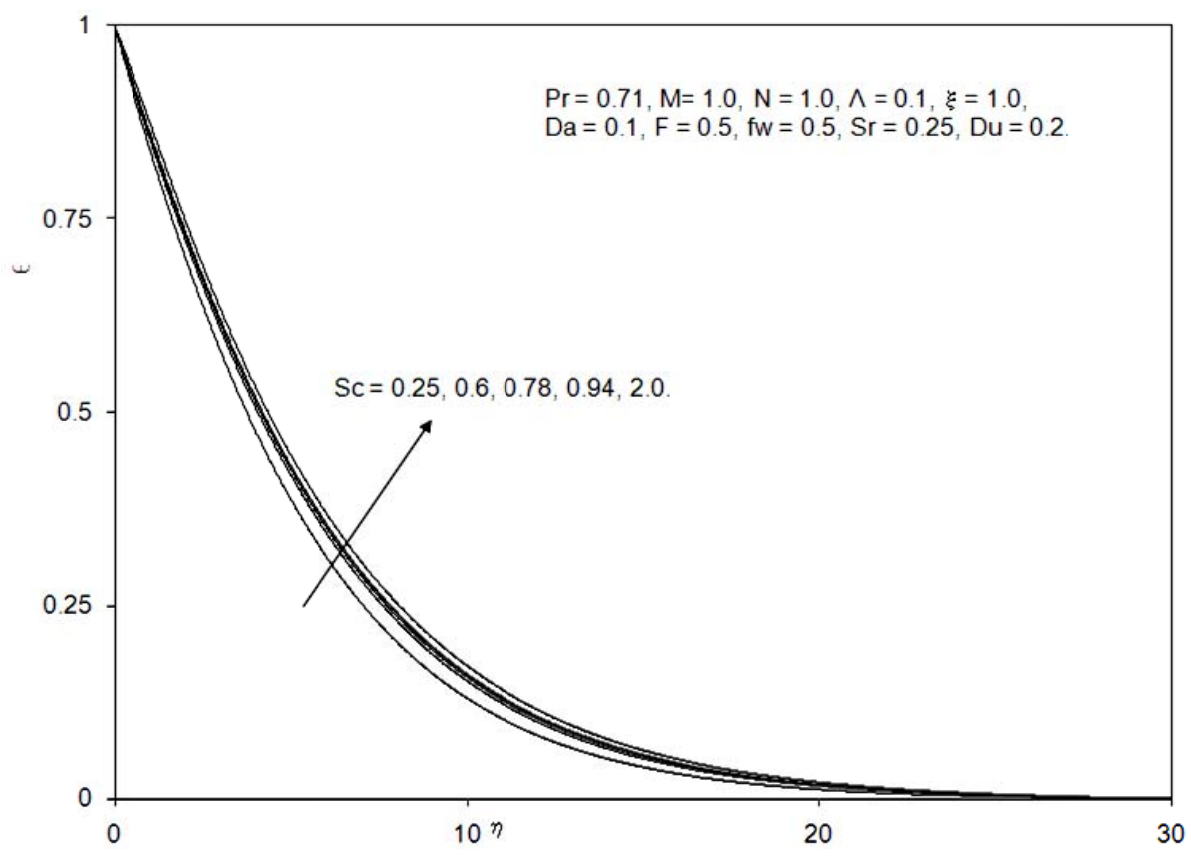

Fig.6b. Effect of the Sc on the temperature profiles.

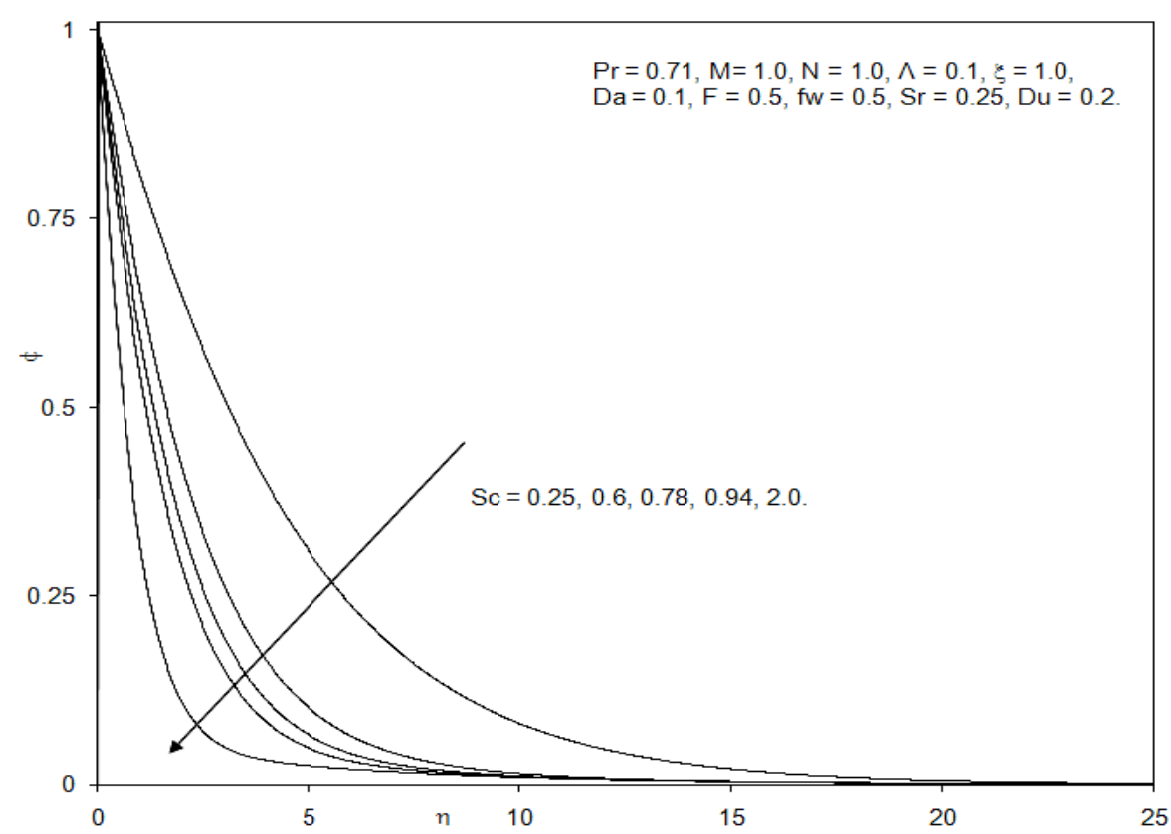

Fig.6c. Effect of the Sc on the concentration profiles.

\section{Conclusions}

Numerical solutions have been presented for the hydromagnetic boundary layer heat and mass transfer flow from a horizontal cylinder immersed in a non-Darcy saturated porous medium with radiation and Soret/Dufour effects. A robust, validated Keller-Box implicit finite difference method has been employed. We use simple central difference derivatives and averages at the mid points of net rectangles to get finite difference equations with a second order truncation error. The procedure includes an implicit finite difference scheme in conjunction with Newton's method for linearization. Time taken for running the program increases with an 
increase in the number of grid points. Increasing the radiation parameter, $F$ decreases velocity and temperature but increases concentration. Increasing the Darcy number, Da increases velocity but reduces temperature and concentration. We noticed that increasing the grid numbers in the computation domain does not change significantly the skin friction, Nusselt and Sherwood coefficients values.

\section{Nomenclature}

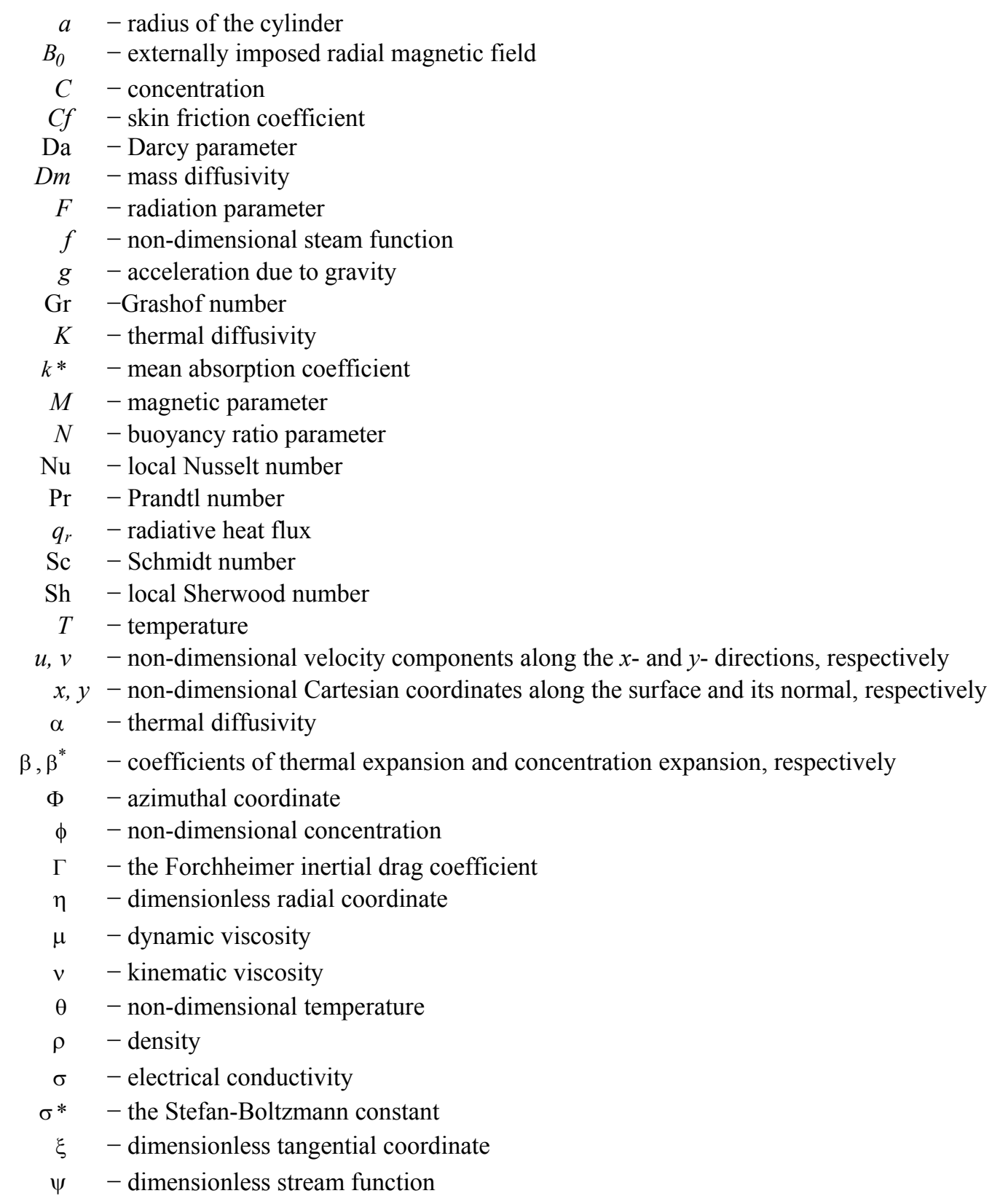

\section{Subscripts}

w - conditions on the wall

$\infty \quad-$ free stream conditions

\section{Superscripts}

'- differentiation with respect to $\eta$ 


\section{References}

[1] Keller H.B. (1970): A new difference method for parabolic problems. - J. Bramble (Editor), Numerical Methods for Partial Differential Equations.

[2] Chiam T.C. (1993): The flat plate magnetohydrodynamic boundary layer flow with a step change in the magnetic field. - J. Phys. Soc. Japan., vol.62, pp.2516-2517.

[3] Rees D.A.S. and Pop I. (1995): Boundary layer flow and heat transfer on a continuous moving wavy surface. Acta Mechanica. vol.112, pp.149-158.

[4] Hossain M.A., Das S.K. and Pop I. (1996): MHD free convection flow near rotating axisymmetric round-nosed bodies. - Magnetohydrodynamics, vol.32, pp.63-67.

[5] Rees D.A.S. and Hossain M.A. (1999): Combined effect of inertia and a spanwise pressure gradient on free convection from a vertical surface in porous media. - Numerical Heat Transfer, Part A: Applications, vol.36, pp.725-736.

[6] Bég O.A., Takhar H.S., Nath G. and Kumari M. (2001): Computational fluid dynamics modeling of buoyancyinduced viscoelastic flow in a porous medium. - Int. J. Applied Mechanics and Engineering, vol.6, pp.187-210.

[7] Bég O.A., Chamkha A.J. and Takhar H.S. (2004): Radiative free convective non-Newtonian fluid flow past a wedge embedded in a porous medium. - Int. J. Fluid Mechanics Research, vol.31, pp.101-115.

[8] Ishak A., Nazar R. and Pop I. (2008): Magnetohydrodynamic (MHD) flow and heat transfer due to a stretching cylinder. - Energy Conversion and Management, vol.49, pp.3265-3269.

[9] Damseh R.A., Tahat M.S. and Benim A.C. (2009): Nonsimilar solutions of magnetohydrodynamic and thermophoresis particle deposition on mixed convection problem in porous media along a vertical surface with variable wall temperature. - Progress in Computational Fluid Dynamics: An International Journal, vol.9, pp.58-65.

[10] Bég O.A., Ramachandra Prasad V., Vasu B., Bhaskar Reddy N., Li Q. and Bhargava R. (2011): Free convection heat and mass transfer from an isothermal sphere to a micropolar regime with Soret/Dufour effects.International Journal of Heat and Mass Transfer, vol.54, No.1-3, pp.9-18.

[11] Prasad V.R., Vasu B., Bég O.A. and Prashad R.D. (2012): Thermal radiation effects on magnetohydrodynamic free convection heat and mass transfer from a sphere in a variable porosity regime. - Communications in Nonlinear Science and Numerical Simulations, vol.17, No.2, pp.654-671.

[12] Gorla R.S.R. and Vasu B. (2016): Unsteady convective heat transfer to a stretching surface in a non-Newtonian nanofluid. - Journal of Nanofluids, vol.5, No.4, pp.581-594.

[13] Gorla R.S.R., Vasu B. and Siddiqa S. (2016): Transient combined convective heat transfer overstretching surface in a non-Newtonian nanofluid using Buongiorno's model. - Journal of Applied Mathematics and Physics (JAMP), vol.4, No.2, pp.443-460.

[14] Roblee L.H.S., Baird R.M. and Tiernery J.W. (1958): Radial porosity variation in packed beds. - AIChem E J., vol.8, pp.359-61.

[15] Vafai K. (1984): Convective flow and heat transfer in variable-porosity media. - J. Fluid Mechanics, vol.147, pp.233-259.

[16] Zueco J., Bég O.A. and Takhar H.S. (2009): Network numerical analysis of magneto-micropolar convection through a vertical circular non-Darcian porous medium conduit. - Computational Materials Science, vol.46, pp.1028-1037.

[17] Minkowycz W.J. and Cheng P. (1976): Free convection about a vertical cylinder embedded in a porous medium. - Int. J. Heat Mass Transfer, vol.19, pp.508-513.

[18] Hamzeh Alkasasbeh T., Mohd Zuki Salleh, Roslinda Nazar and Pop I. (2014): Numerical solutions of radiation effect on magnetohydrodynamic free convection boundary layer flow about a solid sphere with Newtonian heating. - Applied Mathematical Sciences, vol.8, No.140, pp.6989-7000. 
[19] Kumari M. and Gorla R.S.R. (2015): MHD boundary layer flow of a non-Newtonian nanofluid past a wedge. Journal of Nanofluids, vol.4, No.1, pp.73-81(9).

[20] Peri K. Kameswaran, Vasu B., Murthy P.V.S.N. and Gorla R.S.R. (2016): Mixed convection from a wavy surface embedded in a thermally stratified nanofluid saturated porous medium with non-linear Boussinesq approximation. - International Communications in Heat and Mass Transfer, vol.77, pp.78-86.

[21] Beg O.A., Prasad V.R.., Vasu B. and Gorla R.S.R. (2017): Computational modelling of magnetohydrodynamic convection from a rotating cone in orthotropic Darcian porous media. - Journal of the Brazilian Society of Mechanical Sciences and Engineering, vol.39, No.6, pp.2035-2054.

[22] Vasu B., Ram Reddy Ch., Murthy P.V.S.N. and Gorla R.S.R. (2017): Entropy generation analysis in nonlinear convection flow of thermally stratified fluid in saturated porous medium with convective boundary condition. ASME-Journal of Heat Transfer, vol.139, No.9, 091701-1 (10 pages).

[23] Bég O.A., Tasveer A. Bég, Bakier A.Y. and Prasad V. (2009): Chemically-reacting mixed convective heat and mass transfer along inclined and vertical plates with Soret and Dufour effects: Numerical solutions. - Int. J. Applied Mathematics and Mechanics, vol.5, No.2, pp.39-57.

[24] Bhargava R., Sharma R. and Bég O.A. (2009): Oscillatory chemically-reacting MHD free convection heat and mass transfer in a porous medium with Soretand Dufour effects: finite element modeling. - Int. J. Applied Mathematics and Mechanics, vol.5, No.6, pp.15-37.

[25] El-Kabeir S.M.M and Chamkha Ali J. (2013): Heat and mass transfer by mixed convection from a vertical slender cylinder with chemical reaction and Soret and Dufour effects. - Heat Transfer-Asian Research, vol.42, No.7, pp.618-629.

[26] Bhattacharyya K., Layek G.C. and Seth G.S. (2014): Soret and Dufour effects on convective heat and mass transfer in stagnation-point flow towards a shrinking surface. - Phys. Scr., vol.89, No.9, 095203.

[27] Yih K.A. (2000): Effect of uniform blowing/suction on MHD-natural convection over a horizontal cylinder: UWT or UHF. - Acta Mechanica, vol.44, pp.17-27.

[28] Plumb O.A. and Huenefeld T.C. (1981): Non-Darcy natural convection form heated surfaces in porous media. Int. J. Heat Mass Transfer., vol.24, pp.765-768.

[29] Raptis P. and Perdikis C. (2004): Unsteady flow through a highly porous medium in the presence of radiation. Transport Porous Medium J., vol.57, No.2, pp.171-179.

[30] Cebeci T. and Bradshaw P. (1984): Physical and Computational Aspects of Convective Heat Transfer. - New York: Springer.

[31] Vasu B., Prasad V.R. and Bég O.A. (2012): Thermo-diffusion and diffusion-thermo effects on MHD free convective heat and mass transfer from a sphere embedded in a non-Darcian porous medium. - Journal of Thermodynamics, vol.2012, Article ID 725142, 17 pages.

[32] Merkin J.H. (1977): Free convection boundary layers on cylinders of elliptic cross section. - J. Heat Transfer, vol.99, pp.453-457.

[33] Gebhart B. and Pera L. (1971): The nature of vertical natural convection flows resulting from the combined buoyancy effects of thermal and mass diffusion. - Int. J. Heat and Mass Transfer, vol.14, pp.2025-2040.

Received: February 9, 2018

Revised: May 13, 2019 